ARTICLE

\title{
Glucocorticoids suppress inflammation via the upregulation of negative regulator IRAK-M
}

Masanori Miyata ${ }^{1, \star}$, Ji-Yun Lee ${ }^{1, \star}$, Seiko Susuki-Miyata1, Wenzhuo Y. Wang ${ }^{1,2}$, Haidong Xu1, Hirofumi Kai ${ }^{3}$, Koichi S. Kobayashi ${ }^{4}$, Richard A. Flavell ${ }^{5} \&$ Jian-Dong Li ${ }^{1}$

Glucocorticoids are among the most commonly used anti-inflammatory agents. Despite the enormous efforts in elucidating the glucocorticoid-mediated anti-inflammatory actions, how glucocorticoids tightly control overactive inflammatory response is not fully understood. Here we show that glucocorticoids suppress bacteria-induced inflammation by enhancing IRAK-M, a central negative regulator of Toll-like receptor signalling. The ability of glucocorticoids to suppress pulmonary inflammation induced by non-typeable Haemophilus influenzae is significantly attenuated in IRAK-M-deficient mice. Glucocorticoids improve the survival rate after a lethal non-typeable Haemophilus influenzae infection in wild-type mice, but not in IRAK-M-deficient mice. Moreover, we show that glucocorticoids and non-typeable Haemophilus influenzae synergistically upregulate IRAK-M expression via mutually and synergistically enhancing p65 and glucocorticoid receptor binding to the IRAK-M promoter. Together, our studies unveil a mechanism by which glucocorticoids tightly control the inflammatory response and host defense via the induction of IRAK-M and may lead to further development of anti-inflammatory therapeutic strategies.

\footnotetext{
${ }^{1}$ Center for Inflammation, Immunity \& Infection, Institute for Biomedical Sciences, Georgia State University, Atlanta, Georgia 30302, USA. ${ }^{2}$ Department of Microbiology and Immunology, University of Rochester Medical Center, Rochester, New York 14642, USA. ${ }^{3}$ Department of Molecular Medicine, Graduate School of Pharmaceutical Sciences, Kumamoto University, Kumamoto 862-0973, Japan. ${ }^{4}$ Department of Microbial Pathogenesis and Immunology, College of Medicine, Texas A\&M Health Science Center, College Station, Texas 77843, USA. ${ }^{5}$ Department of Immunobiology and Howard Hughes Medical Institute, Yale University School of Medicine, New Haven, Connecticut 06520, USA. * These authors contributed equally to this work. Correspondence and requests for materials should be addressed to J.-D.L. (email: jdli@gsu.edu).
} 
G lucocorticoids (GCs) are the most widely used and most effective treatment to control inflammatory diseases ${ }^{1-3}$. GCs are known to exert their anti-inflammatory effects by binding to glucocorticoid receptors (GRs), leading to the suppression of proinflammatory regulators such as nuclear factor- $\kappa \mathrm{B}(\mathrm{NF}-\mathrm{\kappa} \mathrm{B})$ or activator protein 1 (AP-1) (refs 4,5). Several mechanisms of action of GR have been reported in the past $^{6}$. First, GR binds to p65 and AP-1 to prevent downstream transcription (tethering). Second, GR binds to the glucocorticoid response element (GRE) to initiate the transcription of anti-inflammatory genes (transactivation). Third, negative GRE has been shown to suppress proinflammatory cytokines (transrepression) $)^{7,8}$.

The innate immune and inflammatory response is activated by pattern recognition receptors, including Toll-like receptors (TLRs), on recognition of pathogen-associated molecular patterns ${ }^{9}$. Pattern recognition receptors activate a number of downstream molecules such as tumour necrosis factor (TNF) receptor-associated factor 6 (TRAF6), NF- $\kappa \mathrm{B}$ essential modulator,

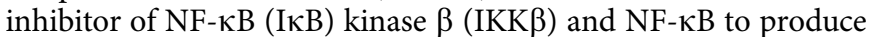
proinflammatory cytokines ${ }^{9}$. Myeloid differentiation factor 88 (MyD88) is a critical downstream adaptor molecule of all TLRs, except TLR3, and interleukin-1 (IL-1) receptor (IL-1R) family (IL-1 $\alpha$, IL-1 $\beta$, IL-18 and IL-33) signalling by recruiting IL-1Rassociated kinase 1 (IRAK1), IRAK4 and TRAF6 (refs 9,10). The studies of MyD88-deficient mice and humans suggest that MyD88 plays a pivotal role in initiating inflammatory responses ${ }^{11,12}$

Negative feedback regulators of inflammation have been recently suggested to play essential roles in tightly controlling inflammatory responses to preserve homeostasis ${ }^{10}$. IRAK-M (also known as IRAK3) is one of the most critical negative feedback regulators of the TLR/IL-1R family signalling via the inhibition of MyD88 and IRAK1/4 activation ${ }^{13-16}$. IRAK-M is a member of the IRAK family and is composed of three conserved domains. However, it does not have any kinase activity due to the lack of a key aspartate in its kinase domain. Thus, IRAK-M has been thought to be a competitor for IRAK1 in associating with MyD88 and TRAF6 (ref. 13). Indeed, IRAK-M-deficient mice exhibited increased inflammatory response in several models ${ }^{15-19}$. IRAK-M expression was initially characterized in monocytes/ macrophages ${ }^{16,20,21}$. Recent studies also demonstrate the expression of IRAK-M in airway epithelial cells ${ }^{20,22}$. Induction of IRAK-M expression by a number of inflammatory stimuli has been shown to suppress inflammation in a negative feedback manner in multiple cell types including macrophages and epithelial cells ${ }^{18,19}$. However, it is unclear if GCs suppress overactive inflammatory responses via induction of negative feedback regulators such as IRAK-M.

In the present study, we show that GCs synergistically enhances IRAK-M expression induced by non-typeable
Haemophilus influenzae (NTHi), not only in airway epithelial cells, but also in macrophages. We found that the overexpression of IRAK-M suppresses, whereas IRAK-M depletion enhances, the NTHi-induced expression of proinflammatory mediators. We further found that IRAK-M-deficiency attenuates the ability of dexamethasone (DEX) to suppress pulmonary inflammation induced by NTHi infection. Lethal NTHi infection-caused mortality was improved by DEX treatment in wild type (WT), but not in IRAK-M-deficient mice. Together our results suggest that the induction of IRAK-M by GCs may be critical to suppress overactive pulmonary inflammatory response in vitro and in vivo. These results thus identified IRAK-M as a novel functional target of GCs.

\section{Results}

GCs synergistically enhance NTHi-induced IRAK-M expression. We sought to determine if GCs regulate the expression of IRAK-M induced by NTHi. Because airway epithelial cells are the front line of defense through initiating inflammatory response, we first examined the effect of GCs on NTHi-induced expression in human respiratory epithelial cells BEAS-2B and A549. DEX synergistically enhanced the NTHi-induced IRAK-M expression at the mRNA level in BEAS-2B and A549 in a dose- and timedependent manner (Fig. 1a,b and Supplementary Fig. 1a,b). Immunoblot analysis revealed that DEX also synergistically enhanced NTHi-induced IRAK-M expression at the protein level (Fig. 1c,d). Moreover, DEX-mediated synergistic enhancement of NTHi-induced IRAK-M expression is also confirmed in primary normal human bronchial epithelial cells (Fig. 1e). We also examined the effect of GCs on IRAK-M expression in macrophages due to their important role in innate immune responses against bacteria ${ }^{23,24}$. As shown in Fig. 1f, the induction of IRAK-M by NTHi and DEX was also observed in primary human monocyte-derived macrophages. Under the same experimental condition, NTHi-induced IL-6 expression was suppressed by DEX (Supplementary Fig. 2). Consistent with these results, DEX also synergistically enhanced the NTHi-induced IRAK-M expression in primary alveolar macrophages of mice (Fig. 1g). Moreover, DEX also synergistically enhanced the NTHi-induced IRAK-M expression at both mRNA and protein levels in lung tissue of mice, as assessed by performing GPCR and immunostaining analyses (Fig. $1 \mathrm{~h}-\mathrm{j}$ ). To further determine which cells express IRAK-M in the lung of mice treated with NTHi and DEX, we next performed immunofluorescence double staining using anti-IRAK-M with anti-E-cadherin or anti-F4/80 antibodies, the marker of epithelial cells and macrophages, respectively. As shown in Fig. 1k, NTHi and DEX markedly induced the expression of IRAK-M in both epithelial cells and macrophages. Of note, IRAK-M-specific staining was completely abolished by an IRAK-M-specific blocking peptide (Fig. 1k).

\footnotetext{
Figure 1 | DEX synergistically enhances the NTHi-induced IRAK-M expression in vitro and in vivo. (a) IRAK-M mRNA expression in human bronchial epithelial BEAS-2B cells treated with DEX $(0.01,0.1,1,10,100$ or 1,000 nM) for $1 \mathrm{~h}$, followed by the stimulation of NTHi for $5 \mathrm{~h}$. (b) IRAK-M mRNA expression in human lung epithelial A549 cells stimulated with DEX (100 nM) for $1 \mathrm{~h}$, followed by NTHi for indicated time. (c) A549 cells were treated with DEX $(100 \mathrm{nM})$ for $1 \mathrm{~h}$, followed by NTHi for $12 \mathrm{~h}$. IRAK-M protein was detected by anti-IRAK-M antibody. (d) IRAK-M protein expression was quantified from three independent experiments. (e) IRAK-M mRNA expression in primary NHBE cells treated with DEX (10, 100 or 1,000 nM) for $1 \mathrm{~h}$ and subjected to NTHi stimulation for $5 \mathrm{~h}$. (f) Human peripheral blood $\mathrm{CD} 14^{+}$monocytes were differentiated to macrophages by culturing them with granulocytemacrophage colony-stimulating factor for 7 days. IRAK-M mRNA was determined after the macrophages were treated with DEX (100 nM) for $1 \mathrm{~h}$, followed by stimulation of NTHi for $5 \mathrm{~h}$. (g) Mice were stimulated with DEX $\left(1 \mathrm{mg} \mathrm{kg}^{-1}\right.$, intraperitoneally) for $2 \mathrm{~h}$ and intratracheally inoculated with NTHi $\left(1 \times 10^{7}\right.$ c.f.u. $)$ for $24 \mathrm{~h}$. The mRNA expression in alveolar macrophages was assessed by qPCR. (h) The IRAK-M mRNA expression in lung was assessed by qPCR. (i) Immunostaining of mouse lung with control IgG or anti-IRAK-M antibody by LSAB (Labelled Streptavidin Biotin) staining system (Scale bar, $50 \mu \mathrm{m}$; magnification, $\times$ 400). (j) Treatment-blind observers scored the IRAK-M expression from the histology results. (k) The lung sections were stained by using indicated antibodies. The arrows and arrowheads indicate the epithelial cells and macrophages merged with IRAK-M expression, respectively. (Scale bar, $50 \mu \mathrm{m}$; magnification, $\times 400$. Data in $\mathbf{a}, \mathbf{b}, \mathbf{d}-\mathbf{h}, n=3 ; \mathbf{j}, n=6$ ) are mean \pm s.d. ${ }^{\star} P<0.05 ;$-test.
} 
Taken together, our data suggest that DEX synergistically enhances NTHi-induced IRAK-M expression at both mRNA and protein levels in vitro and in vivo.

IRAK-M negatively regulates the NTHi-induced inflammation.

To determine the role of the induction of IRAK-M expression in the NTHi-induced inflammatory responses, we established stable BEAS-2B cells expressing IRAK-M (IRAK-M-stable cells; Fig. 2a). As shown in Fig. $2 b$, the NTHi-induced expression of TNF- $\alpha$, IL-1 $\beta$, IL-6, CXCL10 and CCL5 was significantly inhibited in IRAK-M-stable cells as compared with mock cells. Next we sought to confirm the role of endogenous IRAK-M expression in
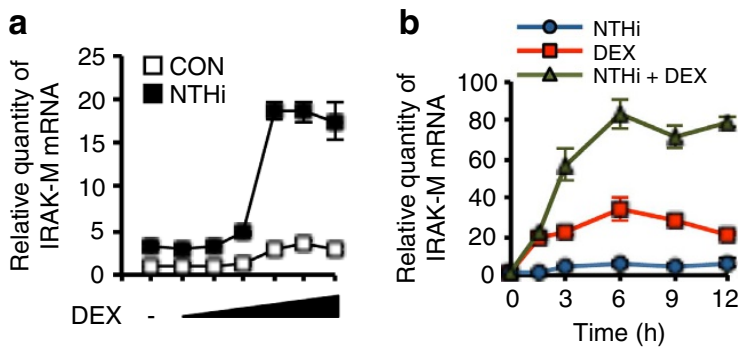

e

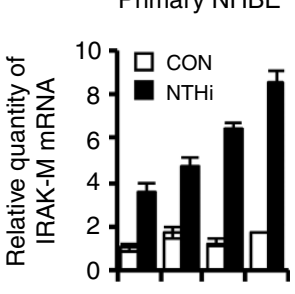

DEX -

\section{f Primary human macrophages}

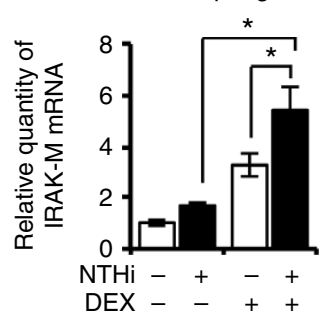

C

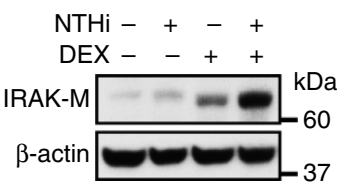

\section{g}

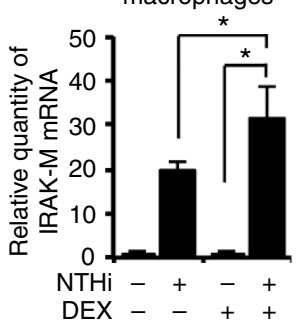

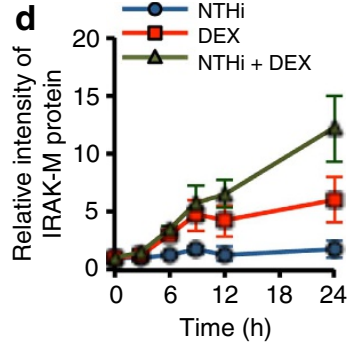

h

Mouse lung

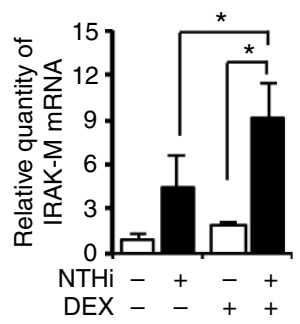

i
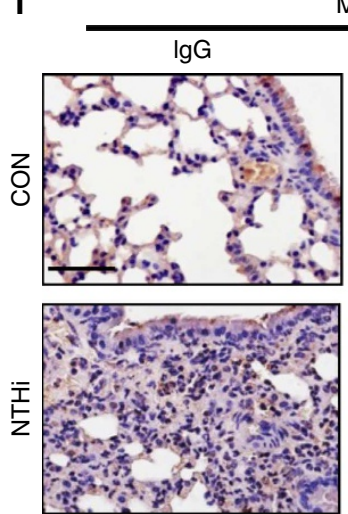

Mock
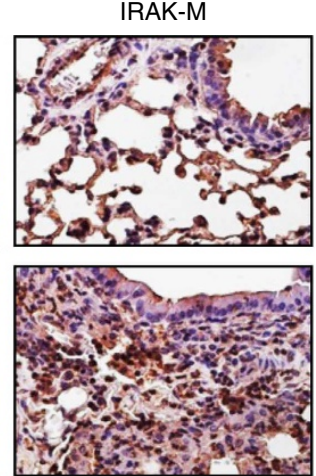

DEX

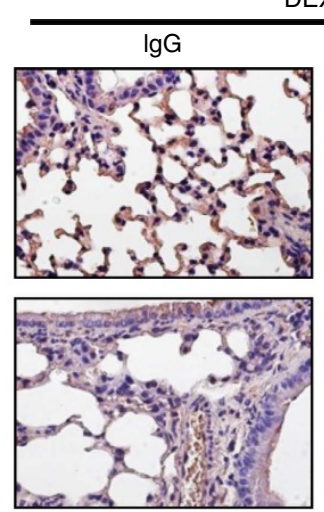

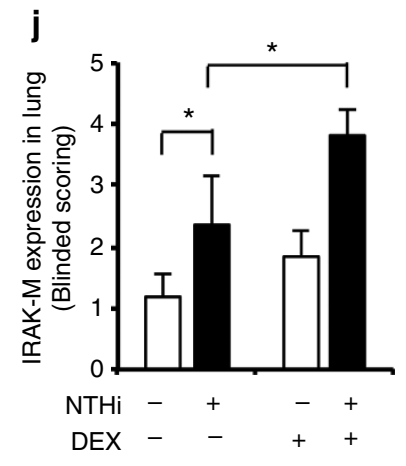

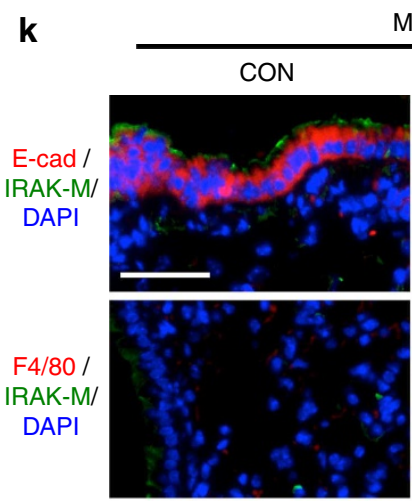

Mock

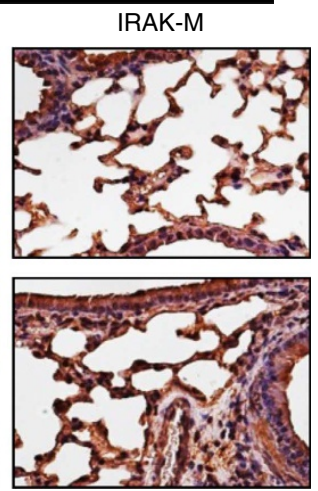

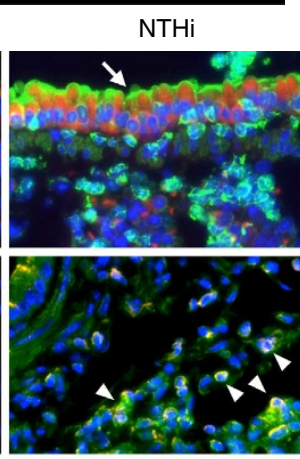

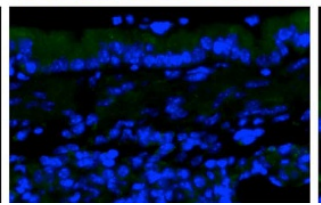

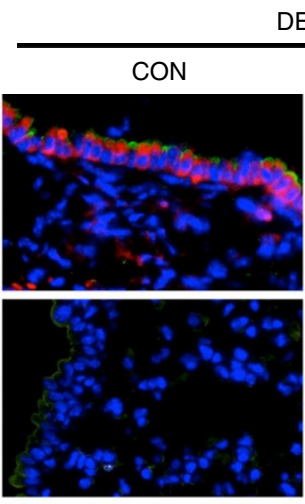

DEX

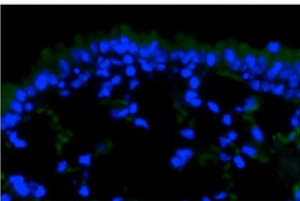

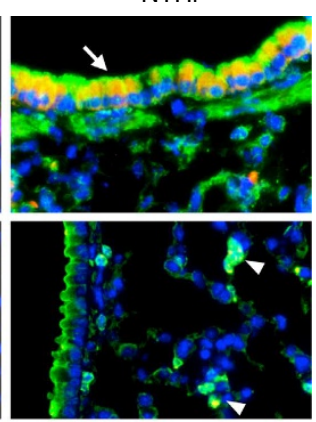

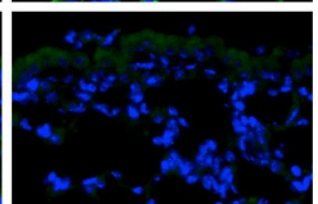


a

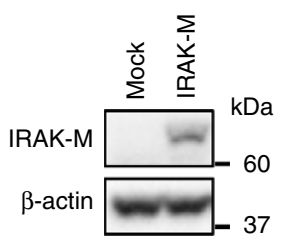

C

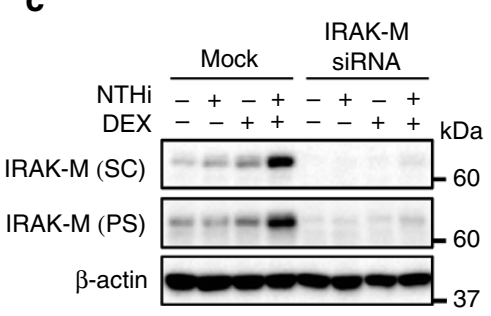

b

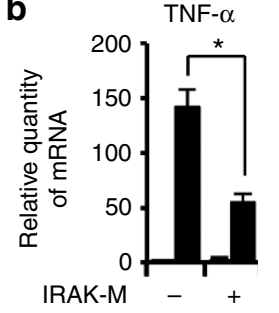

IL-1 $1 \beta$

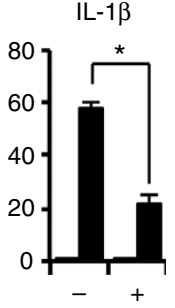

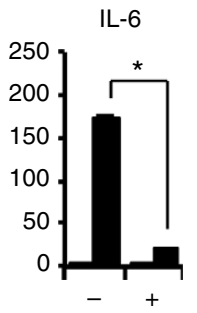
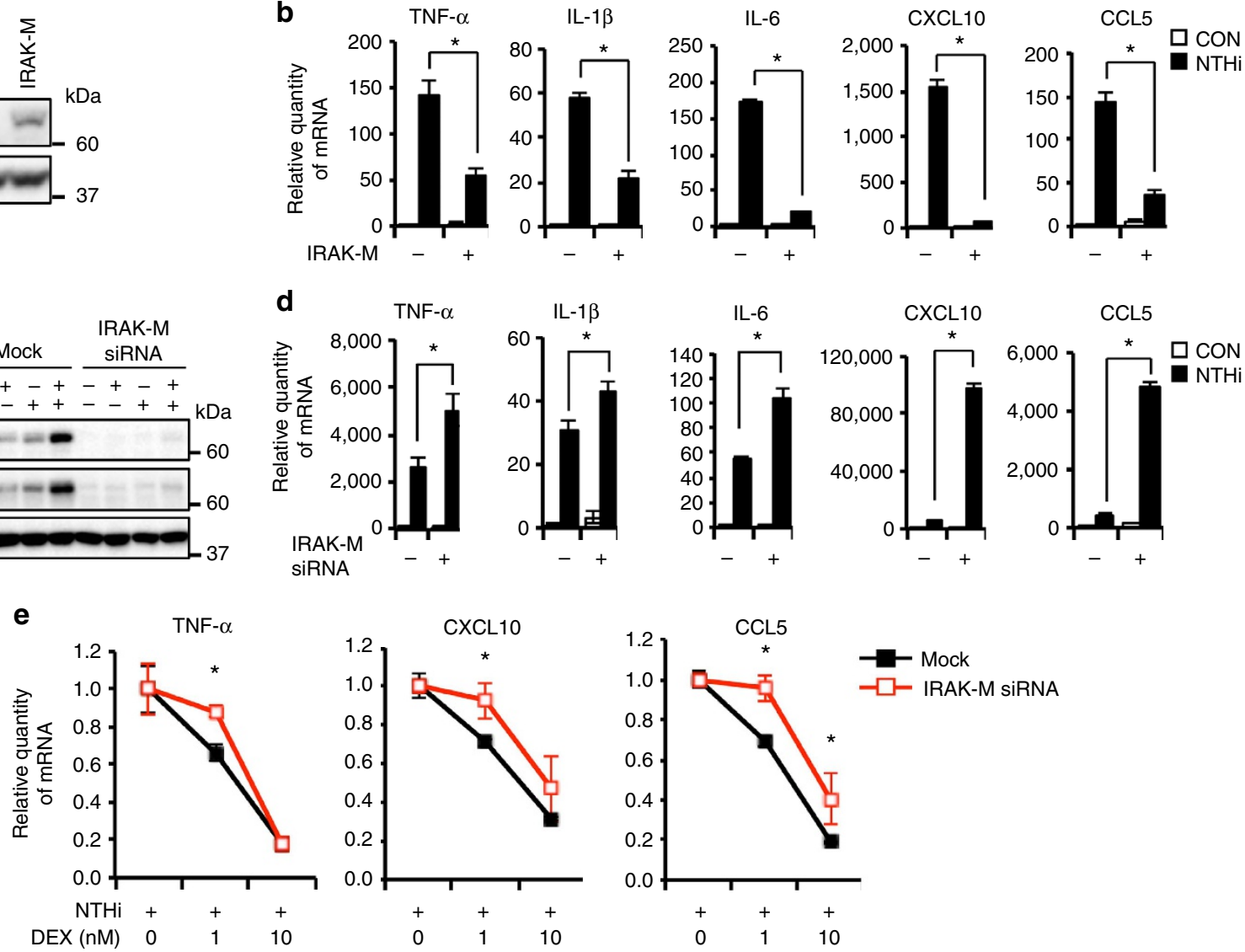

Figure 2 | IRAK-M suppresses the NTHi-induced proinflammatory mediator expression. (a) The proteins from BEAS-2B cells stably overexpressing

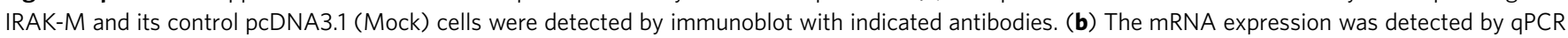
after the stable cells were treated with DEX (100 nM) and NTHi. (c) BEAS-2B cells were transfected with siRNA control or IRAK-M for 72 h. IRAK-M protein expression was detected by immunoblot with anti-IRAK-M antibodies (SC, Santa Cruz Biotechnology sc-100389; PS, ProSci \#2355).

(d) siRNA-transfected BEAS-2B cells were infected with NTHi followed by qPCR. (e) The mRNA level was calculated by following: (NTHi-induced mRNA at each concentration of DEX)/(NTHi-induced mRNA without DEX). Data in $\mathbf{b}, \mathbf{d}, \mathbf{e}, n=3)$ are mean \pm s.d. ${ }^{\star} P<0.05 ; t$-test.

NTHi-induced expression of proinflammatory mediators by depleting IRAK-M using short interfering RNA (siRNA). Immunoblot analysis using two different anti-IRAK-M antibodies revealed that the siRNA depletion of IRAK-M specifically reduced IRAK-M expression (Fig. 2c). IRAK-M depletion markedly enhanced NTHi-induced expression of TNF- $\alpha$, IL-1 $\beta$, IL-6, CXCL10 and CCL5 (Fig. 2d). Moreover, the ability of DEX to suppress these proinflammatory mediators was also significantly attenuated in IRAK-M-depleted cells (Fig. 2e). These data thus provide direct evidence for the role of the induction of IRAK-M expression in NTHi-induced inflammatory responses in the presence of DEX.

GCs suppress inflammation and improve survival via IRAK-M. To determine if DEX suppresses NTHi-induced inflammatory response via the induction of IRAK-M expression, we compared the inhibitory effect of DEX on NTHi-induced innate inflammatory response in WT $\left(\right.$ Irak- $\left.m^{+/+}\right)$with IRAK-M-deficient $\left(\right.$ Irak $\left.-m^{-l^{-}}\right)$mice. We observed that DEX markedly suppressed the NTHi-induced mRNA expression of key proinflammatory mediators including TNF- $\alpha$, IL-1 $\beta$, IL-6, MIP2, CXCL5/LIX, CXCL10 and CCL5, at the mRNA level, in Irak- $m^{+/+}$but not in Irak- $m^{-1}$ mice (Fig. 3a). Enzyme-linked immunosorbent assay analysis confirmed that DEX failed to markedly suppress the NTHi-induced expression of these proinflammatory mediators at the protein level in the bronchoalveolar lavage (BAL) fluid of
Irak- $m^{-1-}$ mice as compared with Irak- $m^{+/+}$mice (Fig. 3b). Consistent with these results, similar results were also observed in mouse alveolar macrophages (Fig. 3c). Moreover, histopathological analysis of the lung of NTHi-infected mice showed that DEX inhibited NTHi-induced leukocyte infiltration in the peribroncheal and interstitial area and also inhibited alveolar wall injury by the inflammatory process in Irak- $m^{+/+}$but not in Irak-m $-1-$ mice (Fig. 4a,b). Similarly, DEX also suppressed NTHi-induced leukocytes infiltration in BAL fluid of $I r a k-m^{+1+}$ mice but not of $I r a k-m^{-1-}$ mice (Fig. 4c). Because an innate inflammatory response initiated by pulmonary epithelial cells is critical for bacterial clearance, we further evaluated the effects of DEX on bacterial clearance in the lungs of $I r a k-m^{-1}$ mice compared with Irak-m $m^{+/+}$mice. DEX treatment increased the colony-formation unit (c.f.u.) of NTHi in the lung of Irak-m $+/+$ mice, but not in the lung of Irak-m-1- mice (Fig. 4d). Consistent with the result of bacterial clearance, DEX also inhibited the NTHi-induced expression of mouse $\beta$-defensin 4 , an orthologue of human BD2, in Irak- $m^{+/+}$mice, but not in Irak- $m^{-1}$ mice (Fig. 4e). Because overactive inflammatory responses contribute significantly to the increased morbidity and mortality in patients with NTHi infections ${ }^{25,26}$, we next evaluated the contribution of IRAK-M in mortality induced by NTHi by infecting mice with a lethal dose of NTHi $\left(5 \times 10^{8}\right.$ c.f.u. $)$ and monitoring survival rate for 7 days. As shown in Fig. 4f, we observed $\sim 50 \%$ mortality in Irak $-\mathrm{m}^{+/+}$mice 7 days after infection. We found no statistically significant difference in 
a
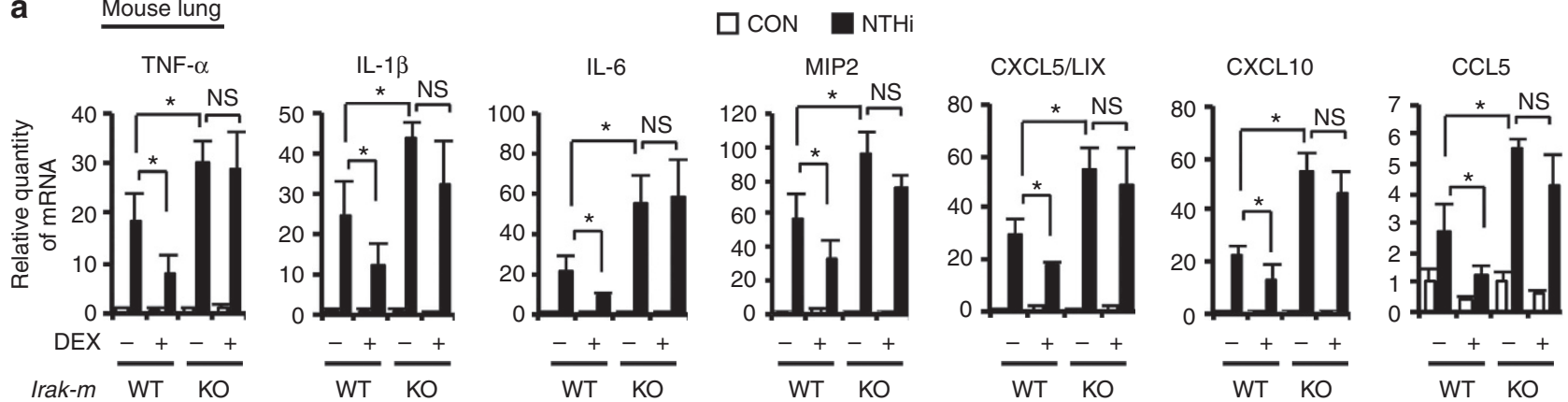

b

Bronchoalveolar lavage (BAL)
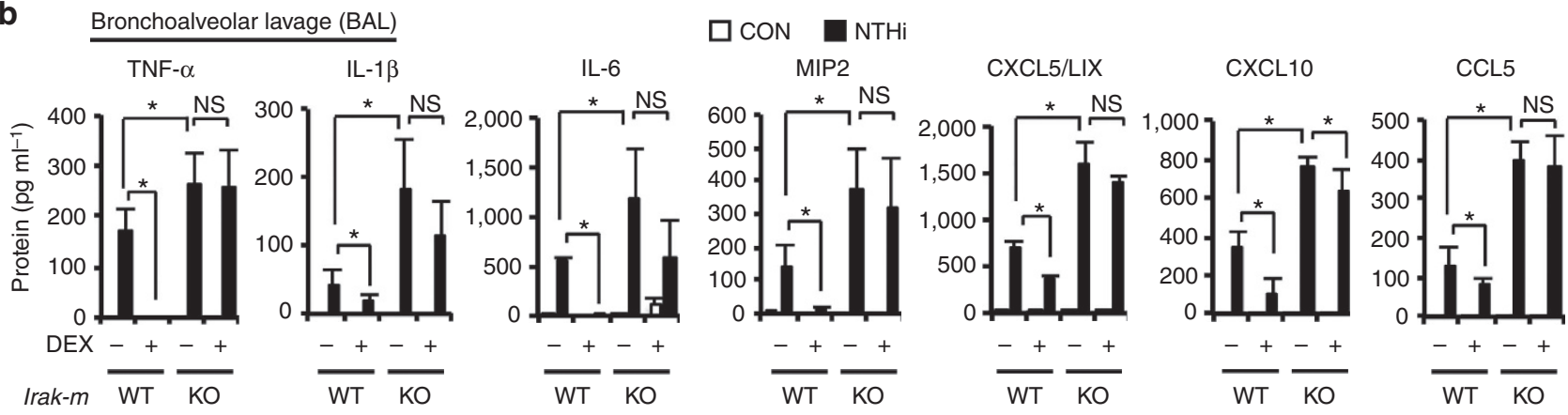

C

\section{Mouse alveolar macrophages}
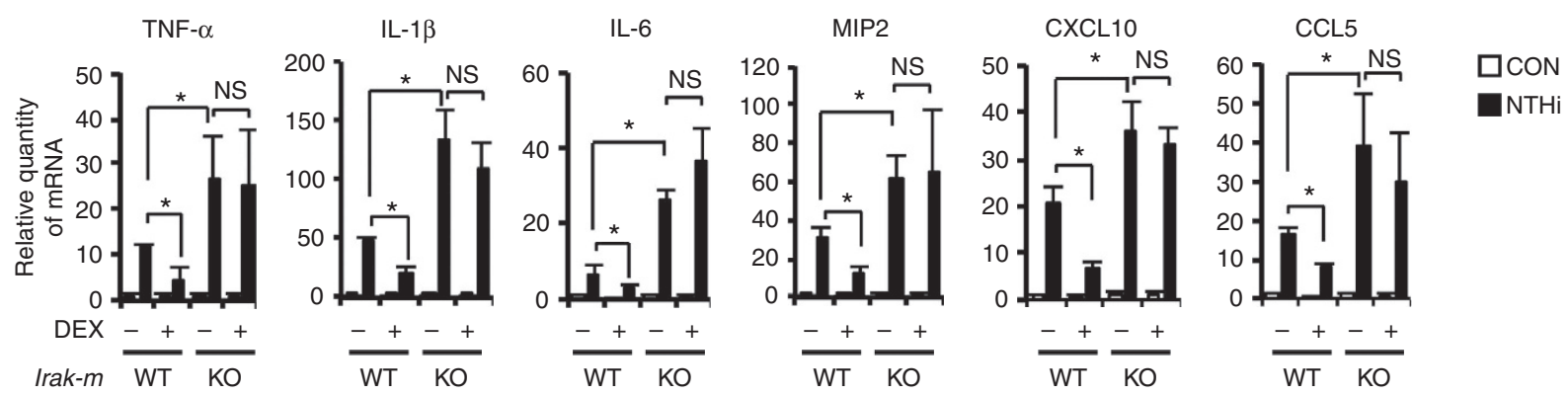

Figure 3 | DEX suppresses the NTHi-induced proinflammatory mediator expression in Irak-m ${ }^{+/+}$but not in Irak- $m^{-/-}$mice. WT and IRAK-M-deficient mice were inoculated with DEX ( $1 \mathrm{mg} \mathrm{kg}^{-1}$, intraperitoneally) for $2 \mathrm{~h}$, followed by intratracheal inoculation with NTHi $\left(1 \times 10^{7} \mathrm{c}\right.$.f.u. per lung $)$ for $24 \mathrm{~h}$. (a) The mRNA expression in the mouse lung tissue was analysed by GPCR. (b) The protein level of proinflammatory mediators in BAL fluid was determined by enzyme-linked immunosorbent assay. (c) The mRNA expression in alveolar macrophages was analysed by $q P C R$. Data in $\mathbf{a}, \mathbf{c}, n=3 ; \mathbf{b}, n=9$ are mean \pm s.d. ${ }^{\star} P<0.05$, NS, non-significant; $t$-test.

survival rate between DEX-untreated Irak-m ${ }^{+/+}$and Irak- $m^{-1-}$ mice $(P=0.362)$. Notably, the survival rate was significantly improved by DEX in Irak-m $m^{+/+}$but not in Irak-m $m^{-/-}$mice (Fig. 4f). Collectively, our data suggest that DEX suppressed bacteria-induced innate inflammatory response and improved survival via the upregulation of IRAK-M expression.

IKK $\beta$ and GR mediate synergistic induction of IRAK-M. Having shown that GCs suppress NTHi-induced inflammatory response via the induction of IRAK-M, the mechanism underlying the regulation of IRAK-M expression still remains unknown. Because NTHi is recognized by TLR2 (refs 27-29), we sought to first evaluate the generalizability of our findings by determining if DEX also enhances the induction of IRAK-M expression by TLR-dependent and -independent inflammatory stimuli. GCs enhanced upregulation of IRAK-M by TNF $\alpha$, IL-1 $\beta$ and Pam3CSK4 in lung epithelial cells (Fig. 5a). Because all of these stimuli that induce IRAK-M, including $\mathrm{NTHi}$, are known to induce inflammatory response via IKK $\beta^{27-29}$, we thus examined if DEX synergistically enhances upregulation of IRAK-M induced by activating IKK $\beta$ signalling through the overexpression of a constitutively active form of IKK $\beta$ (IKK $\beta$ CA). DEX indeed synergistically enhanced the induction of IRAK-M by the direct activation of IKK $\beta$ signalling (Fig. 5b). We further determined if IKK $\beta$ mediates the synergistic upregulation of IRAK-M by DEX and NTHi. Inhibition of IKK $\beta$ using specific inhibitor significantly suppressed the synergistic upregulation of IRAK-M expression at both mRNA and protein levels by DEX and NTHi (Fig. $5 c, d)$, suggesting the requirement of IKK $\beta$ signalling in mediating the synergistic induction of IRAK-M.

Because GR is crucial for GC-mediated biological effects ${ }^{5,6}$, we next assessed the role of GR using RU486 (mifepristone), a GR antagonist. RU486 significantly inhibited the synergistic upregulation of IRAK-M expression at both mRNA and protein levels by DEX and NTHi in BEAS-2B and A549 cells (Fig. 5e,f and Supplementary Fig. 3a). Moreover, GR depletion using siRNA also markedly inhibited the synergistic upregulation of IRAK-M by DEX and NTHi (Fig. 5g and Supplementary Fig. 3b), indicating that GR mediates synergistic induction of IRAK-M. 
a
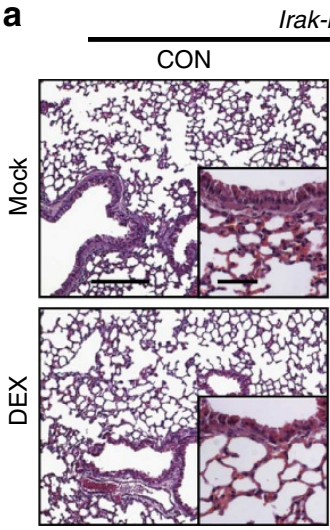

b

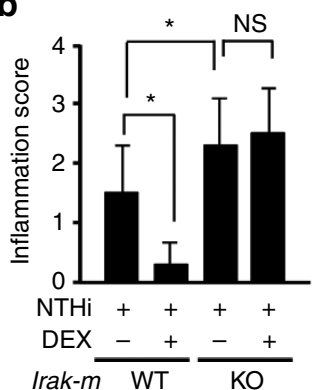

e

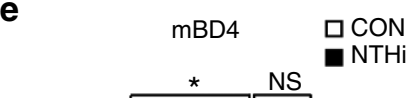

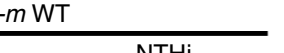
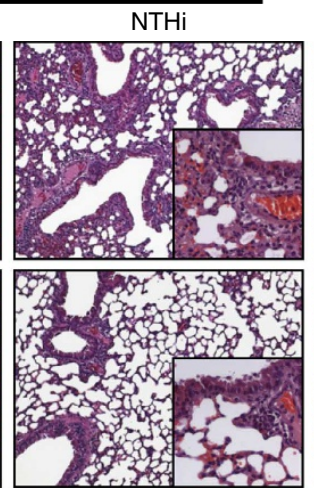

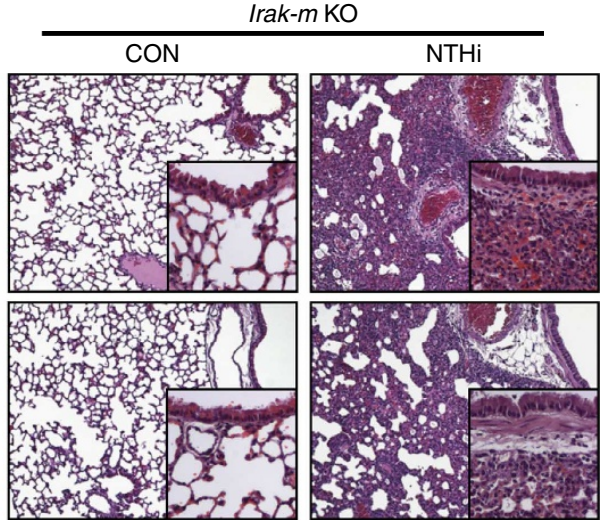

C
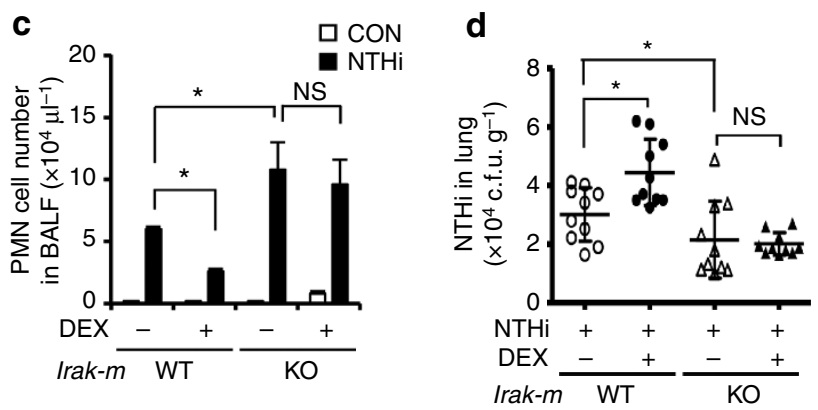

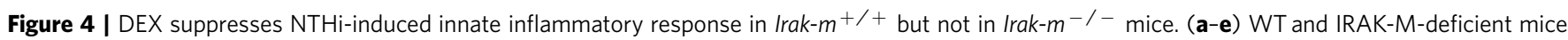
were inoculated with DEX ( $1 \mathrm{mg} \mathrm{kg}^{-1}$, intraperitoneally) for $2 \mathrm{~h}$, followed by intratracheal inoculation with $\mathrm{NTHi}\left(1 \times 10^{7} \mathrm{c} . \mathrm{f}\right.$.u. per lung) for $24 \mathrm{~h}$. (a) Haematoxylin and eosin (H\&E) staining of lung tissues from mice (Scale bar, $200 \mu \mathrm{m}$, magnification, $\times 100$ in large frame; Scale bar $50 \mu \mathrm{m}$, magnification, $\times 400$ in inserted frame). (b) Blinded histopathologic scoring of lung inflammation was performed on H\&E-stained lung sections in a

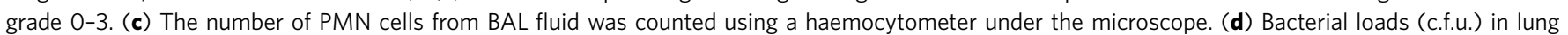
homogenates. (e) The mRNA expression of mBD4 in lung was measured by qPCR (f) WT and IRAK-M-deficient mice were inoculated with DEX ( $1 \mathrm{mg} \mathrm{kg}^{-1}$, intraperitoneally) for $2 \mathrm{~h}$, followed by intratracheal inoculation with lethal dose of NTHi ( $5 \times 10^{8}$ c.f.u. per lung) for 7 days. Survival rate was monitored for indicated days. $P$ values were determined by Kaplan-Meier survival analysis with $\mathrm{GraphPad}$ Prism 5.0 . Data in $\mathbf{b}=8 ; \mathbf{c}, \mathbf{e}, n=3 ; \mathbf{d}=10$, $\mathbf{f}=20$ are mean \pm s.d. ${ }^{\star} P<0.05, N S$, non-significant; $t$-test.

GR is known to regulate gene expression by influencing transcription or mRNA stability ${ }^{30,31}$. Interestingly, the mRNA stability of IRAK-M remained largely unchanged by NTHi or DEX (Supplementary Fig. 4). Consistent with this result, actinomycin D, a transcriptional inhibitor, abrogated the synergistic induction of IRAK-M protein expression induced by DEX and NTHi (Fig. 5h). These data suggest that DEX and NTHi are unable to induce IRAK-M expression in the absence of the on-going transcription.

We sought to further elucidate the molecular mechanism underlying the synergistic induction of IRAK-M by DEX and NTHi. DEX is known to exert its transcriptional activity via the induction of the ligand-dependent dimerization of GR and the subsequent binding of the dimerized GR to GRE in the gene regulatory region of the driven genes ${ }^{4-6}$. We thus first determined if the ligand-induced binding of GR dimer to the GRE in the promoter region of IRAK-M is involved in the synergistic induction of IRAK-M transcription by using a non-steroidal GR-monomer-favouring compound, compound A (CpdA). Unlike DEX, CpdA exhibits no effect on GRE-driven gene transcription $^{32}$. We found that CpdA failed to enhance NTHiinduced expression of IRAK-M (Fig. 5i). In contrast, it still significantly inhibited the NTHi-induced NF- $\kappa$ B activity (Fig. 5j). These data thus suggest that the binding of dimerized GR to GRE is required for the synergistic induction of IRAK-M transcription by DEX and NTHi. 
a
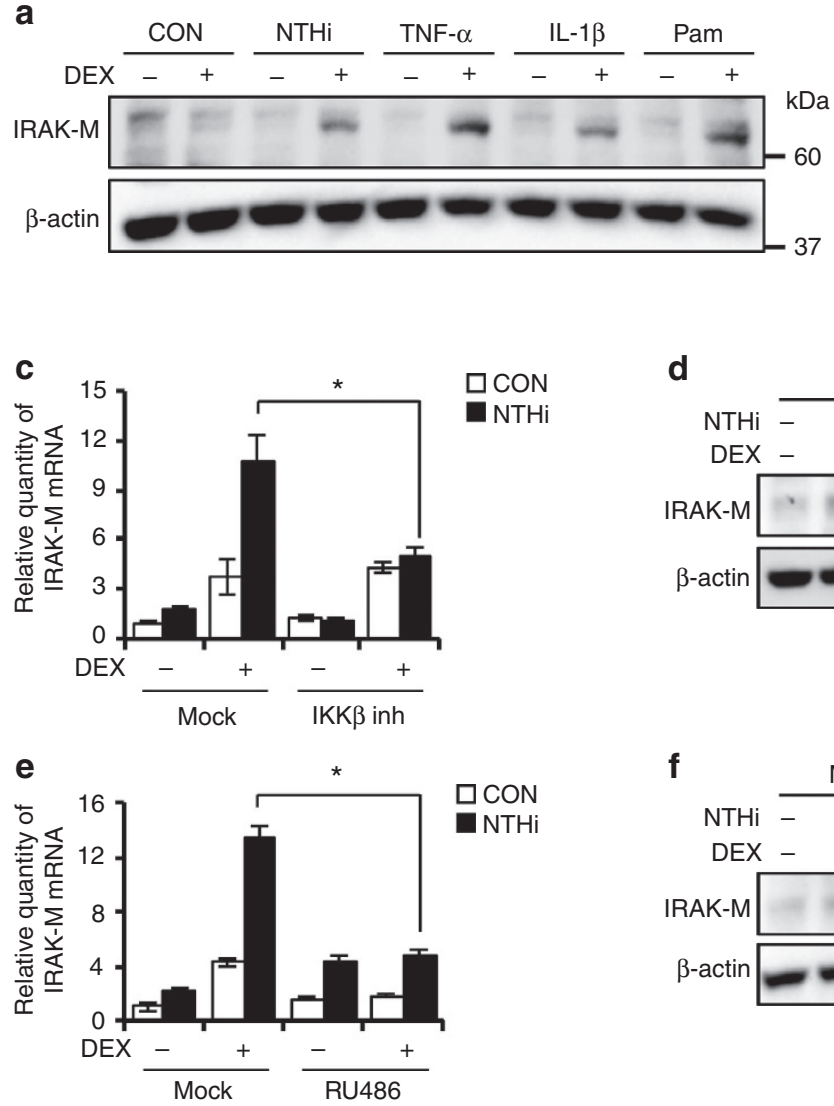

g
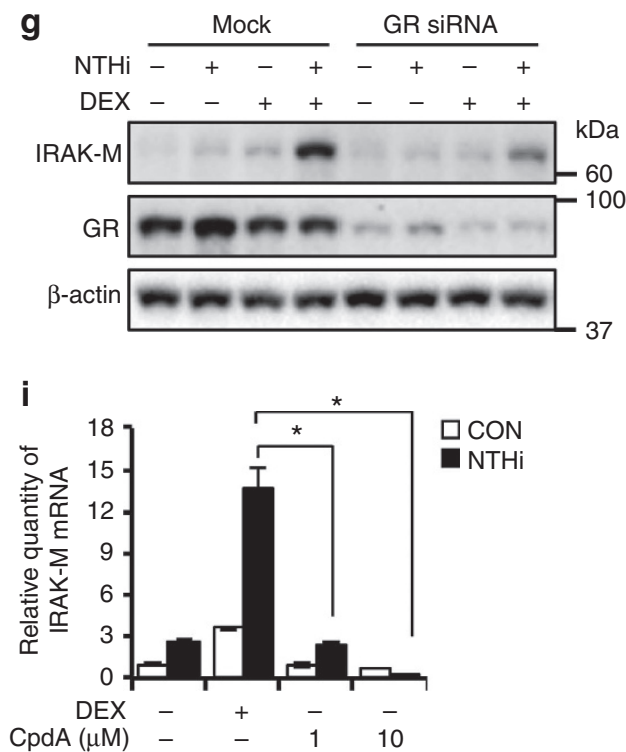

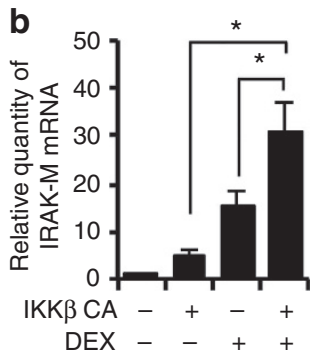

d
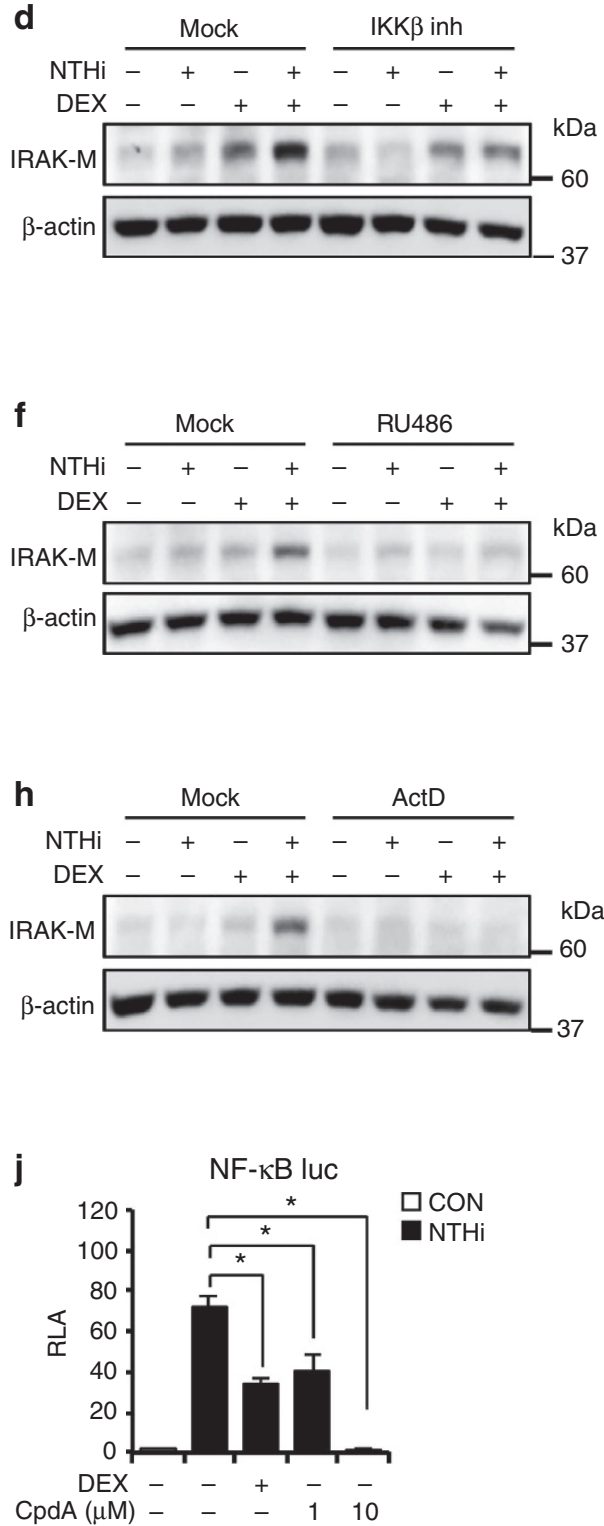

Figure 5 I DEX synergistically enhances the NTHi-induced IRAK-M expression via IKK $\beta$ and GR. (a) Immunoblot shows that IRAK-M protein expression in BEAS-2B cells treated with DEX $(100 \mathrm{nM})$ and NTHi, TNF- $\alpha\left(10 \mathrm{ng} \mathrm{ml}^{-1}\right)$, IL-1 $\beta\left(1 \mathrm{ng} \mathrm{ml}^{-1}\right)$ or Pam3CSK4 $\left(1 \mu \mathrm{g} \mathrm{ml}{ }^{-1}\right)$. (b) IRAK-M mRNA expression assessed by $\mathrm{qPCR}$ in $\mathrm{A} 549$ cells transfected with constitutive active form of IKK $\beta$ (IKK $\beta$ CA) with DEX (100 nM). (c-f), IRAK-M expression in BEAS-2B cells treated with DEX $(100 \mathrm{nM})$ and NTHi, IKK $\beta$ inhibitor $(1 \mu \mathrm{M})(\mathbf{c}, \mathbf{d})$ or RU486 $(1 \mu \mathrm{M} ; \mathbf{e}, \mathbf{f})$. (g) Immunoblot shows IRAK-M protein expression in BEAS-2B cells transfected with siRNA GR. (h) BEAS-2B cells were treated with NTHi, DEX (100 nM) and actinomycin D, ActD $\left(5 \mu \mathrm{g} \mathrm{ml}{ }^{-1}\right)$, followed by immunoblot. (i) The expression of IRAK-M mRNA in BEAS-2B cells treated with DEX (100 nM) and Compound A, CpdA (1 or $10 \mu \mathrm{M})$ followed by NTHi stimulation for $5 \mathrm{~h}$. (j) Relative luciferase activity (RLA) of NF- $\kappa B$ is measured by luciferase assay. BEAS-2B cells transfected with NF- $\kappa B$ luciferase plasmid were treated with CpdA and NTHi. Data $(\mathbf{b}, \mathbf{c}, \mathbf{e}, \mathbf{i}, \mathbf{j}, n=3)$ are mean \pm s.d. ${ }^{\star} P<0.05, \mathrm{NS} ;$-test.

GR and p65 synergistically bind to IRAK-M promoter. We next sought to define the cis-acting DNA elements critical for the synergistic induction of IRAK-M. We constructed a series of deletion mutants in the upstream region of IRAK-M promoter, inserted them to a pGL3 basic vector and measured their luciferase activity. As shown in Fig. 6a, the key cis-acting elements critical for mediating the synergistic induction of IRAK-M transcription resided within the promoter region from -500 to +71 
a

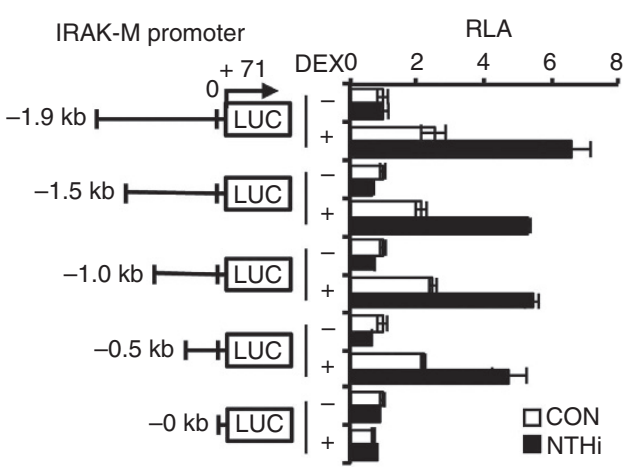

d

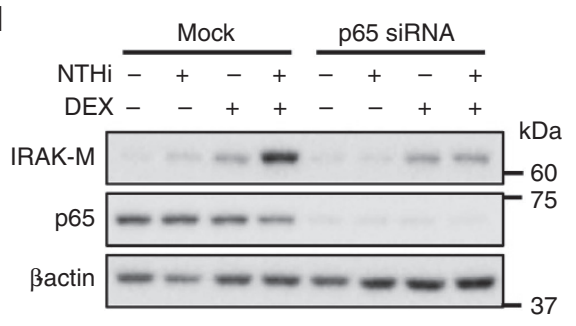

g

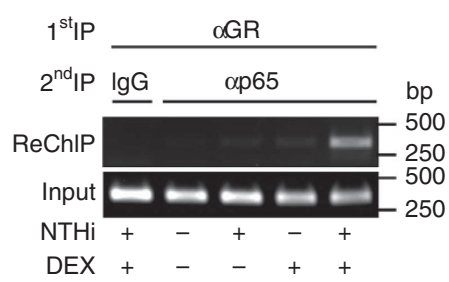

b

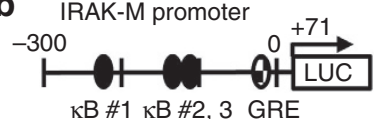

$\kappa B \# 1 \kappa B \# 2,3$ GRE

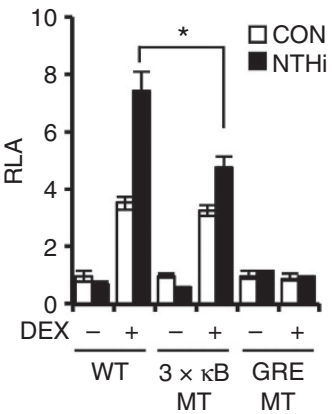

e
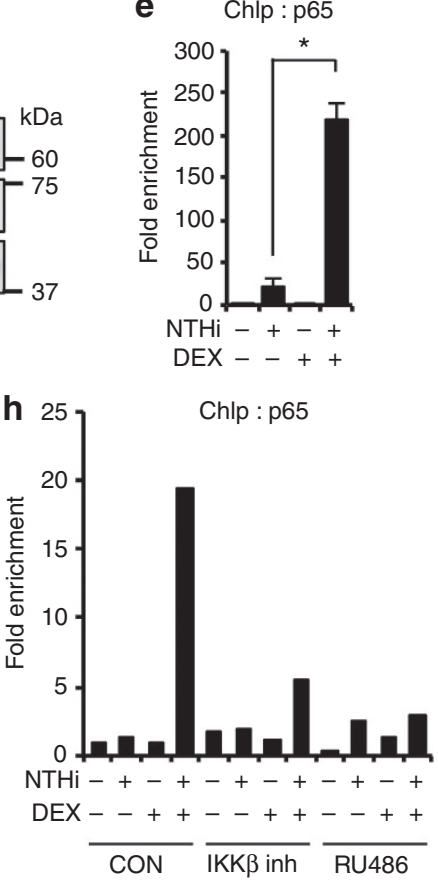

C

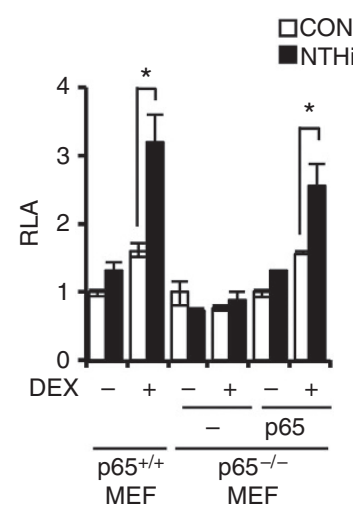

f
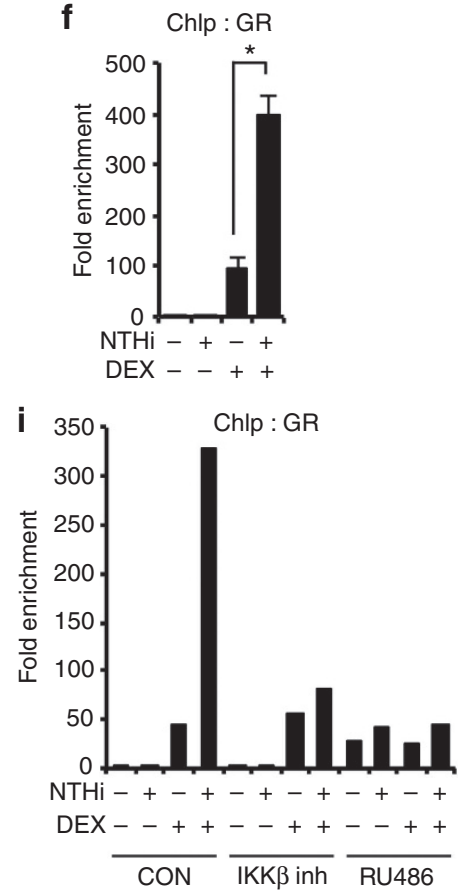

Figure 6 | DEX and NTHi synergistically induce IRAK-M transcription via inducing the binding of p65 and GR to IRAK-M promoter. (a-c) Relative luciferase activity (RLA) was measured after the transfection of IRAK-M promoter in pGL3 basic vector and treated with DEX (100 nM) and NTHi in BEAS-2B cells $(\mathbf{a}, \mathbf{b})$. (c) MEF cells were transfected with IRAK-M-luc ( $-500 \mathrm{bp}$ ), followed by DEX $(1 \mu \mathrm{M})$ and NTHi stimulation. (d) Immunoblot shows IRAK-M protein expression in BEAS-2B cells transfected with siRNA p65. (e,f) ChIP assay in BEAS-2B cells treated with DEX and NTHi for $1 \mathrm{~h}$. IRAK-M promoter regions from -38 to $+39 \mathrm{bp}$ for GRE, from -231 to $-99 \mathrm{bp}$ for $\kappa B$ site were amplified by $\mathrm{qPCR}$. (g) Re-ChIP assay (detailed in Methods). PCR products were detected in agarose gel. (h,i) ChIP assay in BEAS-2B cells treated with IKK $\beta$ inhibitor $(1 \mu \mathrm{M}), \mathrm{RU} 486(1 \mu \mathrm{M}), \mathrm{DEX}(100 \mathrm{nM})$ and NTHi. Data (a-c, $n=3 ; \mathbf{e}, \mathbf{f}, n=2)$ are mean \pm s.d. ${ }^{\star} P<0.05 ;$-test.

base pair (bp). Further in silico sequence analysis revealed the existence of three putative NF- $\kappa \mathrm{B}$-binding sites ( $\kappa \mathrm{B}$ site) and one GRE in the IRAK-M promoter region from -300 to $+71 \mathrm{bp}$ (Supplementary Fig. 5). Interestingly, mutation of this GRE and these three $\kappa \mathrm{B}$ sites markedly inhibited the synergistic activation of IRAK-M promoter by DEX and NTHi (Fig. 6b), thereby further demonstrating the requirement of NF- $\mathrm{KB}$ and GR. Since p65 is a key subunit of $\mathrm{NF}-\kappa \mathrm{B}^{33}$, we next determined if $\mathrm{p} 65$ is critically involved in this synergistic induction of IRAK-M. We observed no synergistic induction of IRAK-M in p65-deficient mouse embryonic fibroblasts (MEFs) treated with NTHi and DEX as compared with WT MEFs, and the reconstitution of p65-deficient MEFs with WT p65 plasmid restored their responsiveness to NTHi and DEX (Fig. 6c). Consistent with these results, p65 knockdown by siRNA inhibited the synergistic induction of IRAK-M expression at the protein level in BEAS-2B cells (Fig. 6d and Supplementary Fig. 6a) and DEX also synergistically enhanced IRAK-M expression induced by expressing WT p65 in BEAS-2B cells (Supplementary Fig. 6b). Taken together, our data suggest that both p65 and GR are required for mediating the synergistic induction of IRAK-M transcription by DEX and NTHi.

We next determined if DEX synergizes with NTHi to induce IRAK-M transcription via synergistically inducing the binding of p65 and GR to IRAK-M promoter region ( $-300 \mathrm{bp}$ to $+71 \mathrm{bp}$ ) containing three $\kappa \mathrm{B}$ sites and GRE. Quantitative analyses using chromatin immunoprecipitation (ChIP) assays revealed that DEX and NTHi synergistically enhanced the binding of both p65 and GR to IRAK-M promoter (Fig. 6e,f). In addition, Re-ChIP assay ${ }^{34}$ revealed that DEX and NTHi induced the interaction of p65 with GR in the context of chromatin (Fig. $6 \mathrm{~g}$ ). Moreover, the synergistic induction of the binding of p65 and GR to IRAK-M 


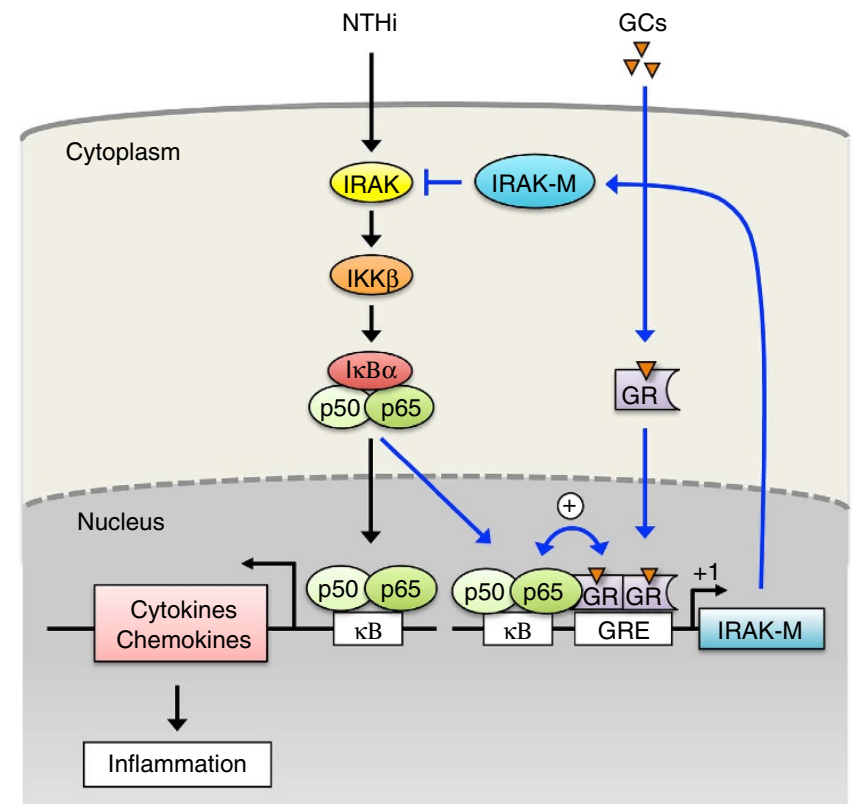

Figure 7 | A schematic representation of suppression of inflammation by GCs via the upregulation of IRAK-M expression.

promoter induced by NTHi and DEX was significantly inhibited by RU486 and IKK $\beta$ inhibitor, respectively (Fig. 6h,i). Together these data suggest that DEX synergizes with NTHi to induce the binding of both GR and p65 to IRAK-M promoter, which, in turn, leads to the induction of IRAK-M transcription in a synergistic manner (Fig. 7).

\section{Discussion}

On infection, an innate inflammatory response can be activated through a TLR. The adaptor proteins IRAK1 and IRAK4 (IRAK1/4) and MyD88 mediate all TLR signalling, except TLR3, in triggering inflammation. It has been demonstrated that MyD88 and IRAK1/4 are critical in activating an inflammatory response in mice $e^{12,35}$ as well as in humans ${ }^{11,36}$. Since GCs have a potent anti-inflammatory effect for multiple usages in a clinical setting, we hypothesized that GCs could target central bottleneck proteins such as MyD88 or IRAK1/4 to tightly regulate inflammatory responses. Here we show that GCs synergistically enhance bacteria-induced IRAK-M, a critical negative regulator of IRAK1/4, in vitro and in vivo. We found that NTHi and GCs cooperatively induced recruitment of p65 and GR to the IRAK-M promoter. In addition, the inhibitory effect of DEX on NTHiinduced inflammatory response is significantly attenuated in mice lacking IRAK-M. Thus, we propose that IRAK-M is a novel functional target of GCs for suppression of bacteria-induced inflammation.

In the present study, we provided experimental evidence for a novel anti-inflammatory mechanisms by which GCs suppress inflammation via the cooperative upregulation of IRAK-M by the GR and NF- $\kappa B$. This finding may be of particular significance as it contradicts a widespread assumption that GCs exert their anti-inflammatory effects principally by antagonizing NF- $\kappa B$ activity ${ }^{4,5}$. If extended to other models of inflammatory diseases, our study may have significant translational implications for design of novel anti-inflammatory agents. Previous studies have predominantly focused on understanding the GC-mediated antiinflammatory actions through the inhibition of positive regulators such as NF-KB and AP-1. Although it has been shown that GCs suppress inflammation by targeting more distal regulators such as I $\mathrm{B} \alpha$ or MKP-1 (refs 37-39), the role of GCs in regulating more proximal and central regulators of inflammation, such as the key bottleneck proteins MyD88 and IRAK1/4, remains unknown. Recently, systematic approaches revealed that MyD88 is a nonredundant core element ${ }^{40,41}$. Owing to GCs' prominence among anti-inflammatory agents, it is logical that GCs may target MyD88-IRAK1/4 to tightly control inflammation. Thus, our study provides novel insight into the tight regulation of MyD88 and IRAK1/4 via modulating the endogenous inhibitor IRAK-M by GCs.

GR has been shown to suppress inflammatory responses via the inhibition of $\mathrm{p} 65$-induced transcription ${ }^{4,37,42}$. A recent genomewide study revealed that GR and p65 mutually enhance their binding to a specific promoter ${ }^{43}$. Thus, the regulation of $\mathrm{p} 65$ - and GR-associated transcription is likely gene specific. Despite previous studies showing that the expression of IRAK-M is induced by inflammatory stimuli in macrophages ${ }^{22,44-47}$ and in osteoblasts $^{48}$, the precise molecular mechanism of IRAK-M regulation at the transcriptional level remains to be understood. We demonstrated that the co-treatment of cells with both NTHi and GCs leads to the synergistic binding of p65 and GR to the IRAK-M promoter. Interestingly, NTHi- and GR-induced binding of $\mathrm{p} 65$ to the IRAK-M promoter is inhibited by the GR antagonist, RU486 (Fig. 6h). Conversely, we also observed a decrease in GR binding in the presence of IKK $\beta$ inhibitor (Fig. 6i). Consistent with previous studies, our data suggest that both p65 and GR synergize with each other to bind to the IRAK-M promoter. These data suggest a novel regulatory mechanism for IRAK-M promoter activity. Future studies investigating how the p65-GR complex activates IRAK-M transcription may lead to a better understanding of the elaborate interplay between bacteria and GCs.

Initially, IRAK-M expression was predominantly characterized in monocytes/macrophages ${ }^{16,20,21}$. Recently it has been shown that during respiratory infections, IRAK-M is expressed and induced, not only in alveolar macrophages, but also in respiratory epithelial cells $22,49,50$. In the present study, we found that GCs synergize with NTHi to induce IRAK-M expression in both respiratory epithelial cells and alveolar macrophages in vitro and in vivo. Importantly, GCs are unable to suppress the NTHiinduced upregulation of proinflammatory mediators in IRAK-Mdepleted lung epithelial cells as well as IRAK-M-deficient alveolar macrophages. Our findings are in line with previous studies demonstrating the expression of IRAK-M in non-myeloid cells including epithelial cells. Thus, it is likely that IRAK-M expressed in both respiratory epithelial cells and macrophages are involved in mediating the ability of GCs to suppress inflammation induced by the respiratory bacterial pathogen, NTHi. It should be noted that our study did not exclude the involvement of other cell types. Future studies using conditional knockout mice with tissue-/cell type-specific deletion of IRAK-M may help determine the contribution of cell-specific induction of IRAK-M expression.

IRAK-M has been shown to act as a critical negative regulator for inflammatory responses. In line with previous findings in pneumonia models ${ }^{15-20,51}$, we observed elevated inflammatory responses in the lung of IRAK-M-deficient mice compared with WT mice. We found that GCs suppressed the NTHi-induced inflammation and significantly improved the survival rate in WT mice but not in IRAK-M-deficient mice (Fig. 4f). Interestingly, no statistically significant difference in survival rate was found between DEX-untreated WT and IRAK-M-deficient mice although IRAK-M-deficient mice show a trend of better 
survival. Our finding is consistent with a recent study showing a reduced lethality in IRAK-M-deficient mice after infection with $S$. pneumonia via the airway ${ }^{20}$. Given that the markers of inflammation are indeed elevated in IRAK-M-deficient mice, it is unclear why IRAK-M-deficient mice did not show an increased mortality. Although the current study does not provide a clear explanation for this apparent anomaly, our preliminary finding on the negative role of IRAK-M in bacteria-induced mucus production may provide possible explanations for this difference. Recently, there is an increasing evidence suggesting that the host has the ability to reduce the tissue damage caused directly by both pathogens and immunopathology through the enhancement of tolerance mechanisms, thus improving host survival ${ }^{52}$. IRAK-M may act as a negative regulator for NTHi-induced host tolerance. Indeed, our preliminary studies revealed that IRAK-M is also a negative regulator for bacteria-induced mucus production, an important mucosal defense mechanism for protecting the host from inflammation- and pathogen-induced tissue damage. Thus, IRAK-M-deficiency results in, not only overactive inflammation, but also overproduced mucus, which are apparently counteractive. This disparity may explain why IRAK-Mdeficient mice did not show an increased mortality. Future studies using mice deficient in mucus production may help to further address this question.

In conclusion, our studies unveil a novel mechanism by which GCs suppress bacteria-induced innate immune and inflammatory responses by upregulating IRAK-M. Our study provides new insights into the previously unidentified role of GCs in suppressing inflammation by targeting the central bottleneck proteins MyD88 and IRAK1/4. It may also lead to the development of new therapeutic strategies to control overactive inflammation.

\section{Methods}

Reagents and antibodies. DEX, Mifepristone (RU486) and Actinomycin D were purchased from Sigma-Aldrich. Compound A was purchased from Enzo Life Science. IKK2 inhibitor IV was purchased from EMD Millipore. Antibodies: GR (sc-8992), p65 (sc-8008, sc-372, sc-109), IRAK-M (sc-100389), $\alpha$-Tubulin (sc-69969), $\beta$-actin (sc-8432), F4/80 (sc-26642), E-cadherin (sc-31020), Donkey anti-rabbit IgG-FITC (sc-2090) and bovine anti-goat IgG-TR (sc-2786) were purchased from Santa Cruz Biotechnology, IRAK-M (\#2355) from ProSci, anti-rabbit HRP-linked antibody (\#7074) and anti-mouse HRP-linked antibody (\#7076) were from Cell Signaling. siRNAs; GR (cat\# L-003424-00-0005,

ON-TARGET plus Human NR3C1 (2908)-SMARTpool, p65 (cat\# L-003533-000005, ON-TARGET plus Human RELA (5970) - SMARTpool) and control siRNA (cat\# D-001810-10-05) were from Thermo Scientific Dharmacon, IRAK-M (IRAK3 (ID 11213) cat\# SR307690 and control siRNA (cat\# SR30004) were from OriGene.

\begin{abstract}
Mice and animal experiments. Irak- $m^{-1-}$ mice have been described previously ${ }^{16}$, and age-matched (8-9 weeks old) male C57BL/6 J mice were used as WT controls. For investigation of the NTHi-induced inflammation in mice, anaesthetized mice were intratracheally inoculated with NTHi at a concentration from $1 \times 10^{7}$ to $5 \times 10^{8}$ c.f.u. per mouse and saline was inoculated as control. The inoculated mice were then killed after NTHi inoculation. For PMN analysis, BAL fluid was collected by cannulating the trachea with sterilized PBS in mice followed by staining with Diff-Quik staining system (modified Giemsa staining). For isolation of macrophages, we collected BAL fluid with $3 \mathrm{ml}$ sterilized PBS. After centrifuge the BAL fluid, macrophages were purified by percoll gradient preparation (Amersham Pharmacia Inc.). For inhibition study, mice were pretreated with DEX $\left(1 \mathrm{mg} \mathrm{kg}^{-1}\right)$ intraperitoneally $2 \mathrm{~h}$ before NTHi inoculation. All animal experiments were approved by the Institutional Animal Care and Use Committee (IACUC) at Georgia State University.
\end{abstract}

Histology and immunostaining. For histological analysis, formalin-fixed paraffinembedded lung tissues were sectioned $(4 \mu \mathrm{m})$ and then stained with haematoxylin and eosin to visualize inflammatory responses and pathological changes in the lung. The stained sections were then imaged and recorded under light systems (AxioVert 40 CFL, AxioCam MRC, and AxioVision LE Image system, Carl Zeiss). The detection of IRAK-M protein was performed using rabbit anti-IRAK-M (ProSci, $2 \mu \mathrm{g} \mathrm{ml}^{-1}$ for Immunohistochemistry, $10 \mu \mathrm{g} \mathrm{ml}^{-1}$ for Immunofluorescence) and Donkey anti-rabbit-FITC (Santa Cruz Biotechnology, $5 \mu \mathrm{g} \mathrm{ml}^{-1}$ ) in the paraffin section of mouse lung tissue. Blocking IRAK-M peptide
(ProSci, cat\# $2355 \mathrm{P}, 25 \mu \mathrm{g} \mathrm{ml}^{-1}$ ) was used for the negative control experiments. Epithelial cells and macrophages were recognized by antibodies of anti-E-cadherin (Santa Cruz Biotechnology, $10 \mu \mathrm{g} \mathrm{ml}^{-1}$ ) and anti-F4/80 (Santa Cruz Biotechnology, $10 \mu \mathrm{g} \mathrm{ml}^{-1}$ ), respectively and followed by bovine anti-goat TR (Santa Cruz Biotechnology, $8 \mu \mathrm{g} \mathrm{ml}^{-1}$ ) incubation. Inflammation score in haematoxylin and eosin staining (Grade; 0 to 3 ) and IRAK-M protein expression intensity score in immunostaining (Grade; 0 to 4 ) were validated in a blinded fashion ${ }^{53-56}$.

Bacterial culture. NTHi strain 12 (also known as R2846) used in this study was a clinically isolated strain that was kindly provided by H. Faden (Children's Hospital of Buffalo, State University of New York, Buffalo, NY) ${ }^{57}$. NTHi were grown on chocolate agar plate at $37^{\circ} \mathrm{C}$ in an atmosphere of $5 \% \mathrm{CO}_{2}$ overnight and inoculated in brain heart infusion broth supplemented with $3.5 \mu \mathrm{g} \mathrm{ml}^{-1} \mathrm{NAD}$ and haemoglobin (BD Biosciences). After overnight incubation, bacteria were subcultured into fresh brain heart infusion and the log phase NTHi, monitored by measurement of optical density $\left(\mathrm{OD}_{600}\right)$ value, was washed and suspended in DMEM for in vitro cell experiments and in isotonic saline for in vivo animal experiments.

Cell culture. All media described below were supplemented with $10 \%$ fetal bovine serum (Sigma-Aldrich). Human airway epithelial A549 cells were maintained in F-12 K media (Gibco), BEAS-2B cells in RPMI 1640 media (Gibco). BEAS-2B cells stably expressing human IRAK-M were obtained by plasmid transfection following geneticin selection $\left(300 \mu \mathrm{g} \mathrm{ml}^{-1}\right)$. Human primary bronchial epithelial (Lonza) cells were maintained in BEGM (bronchial epithelial growth media) supplemented with BEGM SingleQuots. Human peripheral blood CD14 + monocytes (Lonza) were cultured in RPMI 1640 media (Gibco) containing $1 \mathrm{mM}$ pyruvate and GM-CSF (50 $\mathrm{ng} \mathrm{ml}^{-1}$; R\&D systems). MEF cells were obtained from E13 embryos and maintained in DMEM (Corning Cellgro). p65 $5^{-1-}$ MEFs reconstituted with p65 WT were cultured in DMEM (Corning Cellgro) containing puromycin $\left(1.5 \mu \mathrm{g} \mathrm{ml}^{-1}\right)$. All cells were cultured in a humidified atmosphere of $5 \% \mathrm{CO}_{2}$ at $37^{\circ} \mathrm{C}$.

Real-time quantitative RT-PCR analysis. Total RNA was isolated with TRIzol reagent (Life Technologies) by following the manufacturer's instruction. The reverse transcription reaction was performed by using $1 \mu \mathrm{g}$ of RNA in $25 \mu \mathrm{l}$ of reaction buffer of TaqMan reverse transcription reagents (Applied Biosystems) and run under the following protocol: $25^{\circ} \mathrm{C}$ for $10 \mathrm{~min}, 42^{\circ} \mathrm{C}$ for $1 \mathrm{~h}$ and $95^{\circ} \mathrm{C}$ for 5 min (refs 29,58-60). PCR was performed by using Fast SYBR Green Master Mix (Life Technologies). In brief, the reactions were performed in triplicate containing $2 \times$ Universal Master Mix, $1 \mu \mathrm{l}$ of template cDNA, $400 \mathrm{nM}$ primers in a final volume of $12.5 \mu \mathrm{l}$ and they were analysed in a 96-well optical reaction plate (Applied Biosystems). Reactions were amplified under the following protocol: $95^{\circ} \mathrm{C}$ for $20 \mathrm{~s}$ followed by $40 \mathrm{cycle}$ of $95^{\circ} \mathrm{C}$ for $3 \mathrm{~s}$ and $60^{\circ} \mathrm{C}$ for $30 \mathrm{~s}$ and quantified by using StepOnePlus Real-Time PCR System and the manufacturer's corresponding software (StepOnePlus Software v2.3; Applied Biosystems). The relative quantities of mRNAs were obtained by using the comparative Ct method and were normalized using human cyclophilin or mouse glyceraldehydes-3phosphate dehydrogenase as an endogenous control. The primers are described in Supplementary Table 1.

Plasmids and transfections. The expression plasmid of a constitutively active form of IKK $\beta$ (IKK $\beta$-CA, S177E/S181E) was a gift from Dr Anjana Rao (Addgene plasmid \# 11105) (ref. 61). The expression plasmid of p65 was cloned and the insert was transferred to pcDNA3.1 vector (Life Technologies) with BamHI and HindIII sites after we amplified the insert with primers shown in Supplementary Table 2. The luciferase reporter construct of NF- $\kappa B$ contains three copies of the NF- $\kappa \mathrm{B}$ site from the IL-2 receptor $(\alpha)$ promoter by using the following oligonucleotides: 5'-TCGAGACGGCAGGGGAATCTCCCTCTCCG-3' and $3^{\prime}$-CTGCCGTCCCCTTAGAGGGAGAGGCAGCT-5' (refs 27,62). Transient transfections were carried out using TransIT-LT1 reagent (Mirus) or Lipofectamine 2000 (Life Technologies) for plasmid DNA, DharmaFECT4 (Thermo Scientific) for siRNA following the manufacturer's instruction. For cloning of human IRAK-M, we used pDONR223-IRAK3 as a template, which was a gift from Drs William Hahn and David Root (Addgene plasmid \# 23627) (ref. 63). The insert was then transferred to pcDNA3.1 vector (Life Technologies) with XhoI and BamHI site after we amplified the insert with primers shown in Supplementary Table 2. For cloning of IRAK-M promoter, we used BAC clone RP11-937C6 (BACPAC Resources Center, Children's Hospital Oakland Research Institute) as a template. PCR was performed using primers shown in Supplementary Table 2. PCR products were transferred into pGL3 basic vector (Promega) with MluI and XhoI site. The sequences were verified from at least three clones. The GRE and NF- $\kappa B$ mutants of IRAK-M promoter were constructed by using the QuikChange II site-directed mutagenesis kit (Agilent Technologies) with primers shown in Supplementary Table 2.

Western blot analysis. Western blots were performed using whole-cell extracts in

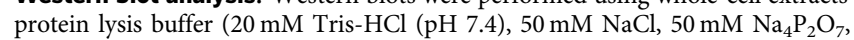


$30 \mathrm{mM} \mathrm{NaF}, 5 \mu \mathrm{M} \mathrm{ZnCl}_{2}$, $2 \mathrm{mM}$ Iodoacetic acid, $1 \%$ Triton-X) with freshly added $1 \mathrm{mM}$ sodium orthovanadate and protease inhibitor cocktail (Sigma-Aldrich), separated on $8 \%$ SDS-polyacrylamide gel electrophoresis gels and transferred to polyvinylidene difluoride membranes. The membrane was blocked with $5 \%$ non-fat dry milk in Tris-buffered saline (TBS) containing $0.1 \%$ Tween 20 (TBS-T). The membrane was then incubated in a 1:2,000 dilution of a primary antibody in 5\% bovine serum albumin-TBS-T at room temperature for $1 \mathrm{~h}$ or at $4{ }^{\circ} \mathrm{C}$ for $16 \mathrm{~h}$. After washing three times with TBS-T, the membrane was incubated with 1:10,000 dilution of the corresponding secondary antibody in $2.5 \%$ non-fat dry milk-TBS-T at room temperature for $2 \mathrm{~h}$. Respective proteins were developed by using Amersham ECL Prime Regent (GE Healthcare Biosciences) and image were obtained by ChemiDoc XRS + System. Images have been cropped for presentation. Full-size images are presented in Supplementary Figs 7-9.

Chromatin immunoprecipitation. ChIP assay was performed with minor modifications of the previous study ${ }^{42,64}$. The cells were crosslinked by incubation with $1 \%$ formaldehyde at room temperature for $10 \mathrm{~min}$, followed by incubation with $0.125 \mathrm{M}$ glycine for $5 \mathrm{~min}$. Cells were washed twice with ice-cold PBS and lysed with cell lysis buffer (50 mM HEPES (pH7.4), $1 \mathrm{mM}$ EDTA, $85 \mathrm{mM} \mathrm{KCl,} \mathrm{10 \%}$ glycerol, $0.5 \% \mathrm{NP} 40$, supplemented with protease inhibitor cocktail). Nuclei was collected by centrifugation at $850 \mathrm{~g}$ for $5 \mathrm{~min}$ and suspended in Nuclei lysis buffer (50 mM Tris- $\mathrm{HCl}$ (pH 8.0), $2 \mathrm{mM}$ EDTA, $150 \mathrm{mM} \mathrm{NaCl}, 5 \%$ glycerol, $1 \%$ Triton$\mathrm{X}-100,0.1 \%$ SDS, supplemented with protease inhibitor cocktail). The chromatin was sheared by sonication (Branson digital sonicator) to an average size $500 \mathrm{bp}$ and precleared with Protein G PLUS-Agarose (Santa Cruz Biotechnology) or Dynabeads protein $\mathrm{G}$ (Life Technologies). The precleared chromatin was incubated with $5 \mu \mathrm{g}$ of primary antibodies overnight at $4^{\circ} \mathrm{C}$, followed by incubation with Protein G PLUS-Agarose or Dynabeads protein G for $2 \mathrm{~h}$. The immunoprecipitates were washed two times with ChIP wash buffer I ( $20 \mathrm{mM}$ Tris- $\mathrm{HCl}(\mathrm{pH} 8.0)$, $150 \mathrm{mM} \mathrm{NaCl}, 1 \%$ Triton-X-100, $0.1 \%$ SDS, $2 \mathrm{mM}$ EDTA), two times with ChIP wash buffer II (20 mM Tris- $\mathrm{HCl}$ (pH 8.0), $500 \mathrm{mM} \mathrm{NaCl}, 1 \%$ Triton-X-100, $0.1 \%$ SDS, $2 \mathrm{mM}$ EDTA), one time with ChIP wash buffer III ( $20 \mathrm{~m}$ Tris- $\mathrm{HCl}$ (pH 8.0), $150 \mathrm{mM} \mathrm{NaCl}, 500 \mathrm{mM} \mathrm{LiCl}, 1 \% \mathrm{NP} 40,1 \%$ deoxycholate, $1 \mathrm{mM}$ EDTA) and two times with TE buffer ( $10 \mathrm{mM}$ Tris- $\mathrm{HCl}$ ( $\mathrm{pH}$ 8.0), $1 \mathrm{mM}$ EDTA). The precipitated chromatin complexes were eluted in elution buffer ( $\left.1 \% \mathrm{SDS}, 0.1 \mathrm{M} \mathrm{NaHCO}_{3}\right)$ at room temperature for $30 \mathrm{~min}$ with vortex every $5 \mathrm{~min}$. After reverse crosslink at $65{ }^{\circ} \mathrm{C}$ for $18 \mathrm{~h}$ and protein digestion with Proteinase $\mathrm{K}$ (Thermo Scientific) at $55^{\circ} \mathrm{C}$ for $3 \mathrm{~h}$, DNA was isolated using MiniElute PCR purification kit (Quiagen) with $30 \mu \mathrm{l}$ elution with UltraPure DNAase/RNAase-Free Distilled Water (Life Technologies). PCR was performed with primers shown in Supplementary Table 2.

Re-ChIP. Re-ChIP assay was performed as described previously ${ }^{34}$. In brief, crosslinking was performed using $2 \mathrm{mM}$ disuccinimidyl glutarate (Thermo Scientific) for $45 \mathrm{~min}$ followed by $1 \%$ formaldehyde for $10 \mathrm{~min}$ at room temperature. After the first IP, immune complexes were eluted in Re-ChIP elution buffer (10 mM Tris-HCl (pH 8.0), 2 mM EDTA, 2\% SDS, 15 mM DTT) including protease inhibitor cocktail at room temperature for $30 \mathrm{~min}$. The elution was diluted 20 times with ChIP dilution buffer ( $16.7 \mathrm{mM}$ Tris- $\mathrm{HCl}(\mathrm{pH} 8.0), 167 \mathrm{mM} \mathrm{NaCl}$, $1.2 \mathrm{mM}$ EDTA, $1.1 \%$ Triton-X) supplemented with protease inhibitor cocktail, $100 \mu \mathrm{g} \mathrm{ml}^{-1}$ bovine serum albumin , $100 \mu \mathrm{g} \mathrm{ml}^{-1}$ salmon sperm DNA, followed by second IP. The second IP samples were washed, eluted and purified as described above ChIP procedure. PCR was performed with $1 \mu \mathrm{l}$ purified DNA by using PrimeSTAR Max DNA polymerase (Takara) with primers IRAK-M $-300 \mathrm{bp}$ forward and IRAK-M 0 bp reverse shown in Supplementary Table 2.

Statistical analysis. Data are shown as mean \pm s.d. Statistical analysis was assessed by $t$-test. $P<0.05$ was considered statistically significant.

\section{References}

1. Quax, R. A. et al. Glucocorticoid sensitivity in health and disease. Nat. Rev. Endocrinol. 9, 670-686 (2013).

2. Clark, A. R. \& Belvisi, M. G. Maps and legends: the quest for dissociated ligands of the glucocorticoid receptor. Pharmacol. Ther. 134, 54-67 (2012).

3. Beck, I. M. et al. Crosstalk in inflammation: the interplay of glucocorticoid receptor-based mechanisms and kinases and phosphatases. Endocr. Rev. 30, 830-882 (2009).

4. Luecke, H. F. \& Yamamoto, K. R. The glucocorticoid receptor blocks P-TEFb recruitment by NFkappaB to effect promoter-specific transcriptional repression. Genes Dev. 19, 1116-1127 (2005).

5. Busillo, J. M. \& Cidlowski, J. A. The five Rs of glucocorticoid action during inflammation: ready, reinforce, repress, resolve, and restore. Trends Endocrinol. Metab. 24, 109-119 (2013).

6. Kadmiel, M. \& Cidlowski, J. A. Glucocorticoid receptor signaling in health and disease. Trends Pharmacol. Sci. 34, 518-530 (2013).

7. Surjit, M. et al. Widespread negative response elements mediate direct repression by agonist-liganded glucocorticoid receptor. Cell 145, 224-241 (2011).
8. Uhlenhaut, N. H. et al. Insights into negative regulation by the glucocorticoid receptor from genome-wide profiling of inflammatory cistromes. Mol. Cell 49, 158-171 (2013).

9. Kawai, T. \& Akira, S. The role of pattern-recognition receptors in innate immunity: update on Toll-like receptors. Nat. Immunol. 11, 373-384 (2010).

10. Kondo, T., Kawai, T. \& Akira, S. Dissecting negative regulation of Toll-like receptor signaling. Trends Immunol. 33, 449-458 (2012).

11. von Bernuth, H. et al. Pyogenic bacterial infections in humans with MyD88 deficiency. Science 321, 691-696 (2008).

12. Adachi, O. et al. Targeted disruption of the MyD88 gene results in loss of IL-1- and IL-18-mediated function. Immunity 9, 143-150 (1998).

13. Janssens, S. \& Beyaert, R. Functional diversity and regulation of different interleukin-1 receptor-associated kinase (IRAK) family members. Mol. Cell 11, 293-302 (2003).

14. Hassan, F. et al. Involvement of interleukin-1 receptor-associated kinase (IRAK)-M in toll-like receptor (TLR) 7-mediated tolerance in RAW 264.7 macrophage-like cells. Cell. Immunol. 256, 99-103 (2009).

15. Deng, J. C. et al. Sepsis-induced suppression of lung innate immunity is mediated by IRAK-M. J. Clin. Invest. 116, 2532-2542 (2006).

16. Kobayashi, K. et al. IRAK-M is a negative regulator of Toll-like receptor signaling. Cell 110, 191-202 (2002).

17. Berglund, M. et al. IL-1 receptor-associated kinase M downregulates DSS-induced colitis. Inflamm. Bowel Dis. 16, 1778-1786 (2010).

18. Standiford, T. J. et al. TGF-beta-induced IRAK-M expression in tumorassociated macrophages regulates lung tumor growth. Oncogene 30, 2475-2484 (2011).

19. Sumpter, T. L. et al. DAP12 promotes IRAK-M expression and IL-10 production by liver myeloid dendritic cells and restrains their $\mathrm{T}$ cell allostimulatory ability. J. Immunol. 186, 1970-1980 (2011).

20. van der Windt, G. J. et al. Interleukin 1 receptor-associated kinase $m$ impairs host defense during pneumococcal pneumonia. J. Infect. Dis. 205, 1849-1857 (2012).

21. Wesche, H. et al. IRAK-M is a novel member of the Pelle/interleukin-1 receptor-associated kinase (IRAK) family. J. Biol. Chem. 274, 19403-19410 (1999).

22. Lagler, H. et al. TREM-1 activation alters the dynamics of pulmonary IRAK-M expression in vivo and improves host defense during pneumococcal pneumonia. J. Immunol. 183, 2027-2036 (2009).

23. Punturieri, A., Copper, P., Polak, T., Christensen, P. J. \& Curtis, J. L. Conserved nontypeable Haemophilus influenzae-derived TLR2-binding lipopeptides synergize with IFN-beta to increase cytokine production by resident murine and human alveolar macrophages. J. Immunol. 177, 673-680 (2006).

24. Wieland, C. W. et al. The MyD88-dependent, but not the MyD88-independent, pathway of TLR4 signaling is important in clearing nontypeable haemophilus influenzae from the mouse lung. J. Immunol. 175, 6042-6049 (2005).

25. Decramer, M., Janssens, W. \& Miravitlles, M. Chronic obstructive pulmonary disease. Lancet 379, 1341-1351 (2012).

26. Sethi, S. \& Murphy, T. F. Infection in the pathogenesis and course of chronic obstructive pulmonary disease. N. Engl. J. Med. 359, 2355-2365 (2008).

27. Shuto, T. et al. Activation of NF-kappa B by nontypeable Hemophilus influenzae is mediated by toll-like receptor 2-TAK1-dependent NIK-IKK alpha /beta-I kappa B alpha and MKK3/6-p38 MAP kinase signaling pathways in epithelial cells. Proc. Natl Acad. Sci. USA 98, 8774-8779 (2001).

28. Watanabe, T., Jono, H., Han, J., Lim, D. J. \& Li, J. D. Synergistic activation of NF-kappaB by nontypeable Haemophilus influenzae and tumor necrosis factor alpha. Proc. Natl Acad. Sci. USA 101, 3563-3568 (2004).

29. Jono, H. et al. NF-kappaB is essential for induction of CYLD, the negative regulator of NF-kappaB: evidence for a novel inducible autoregulatory feedback pathway. J. Biol. Chem. 279, 36171-36174 (2004).

30. Dhawan, L., Liu, B., Blaxall, B. C. \& Taubman, M. B. A novel role for the glucocorticoid receptor in the regulation of monocyte chemoattractant protein-1 mRNA stability. J. Biol. Chem. 282, 10146-10152 (2007).

31. Gille, J., Reisinger, K., Westphal-Varghese, B. \& Kaufmann, R. Decreased mRNA stability as a mechanism of glucocorticoid-mediated inhibition of vascular endothelial growth factor gene expression by cultured keratinocytes. J. Invest. Dermatol. 117, 1581-1587 (2001).

32. Robertson, S. et al. Abrogation of glucocorticoid receptor dimerization correlates with dissociated glucocorticoid behavior of compound a. J. Biol. Chem. 285, 8061-8075 (2010).

33. Baldwin, Jr. A. S. The NF-kappa B and I kappa B proteins: new discoveries and insights. Annu. Rev. Immunol. 14, 649-683 (1996).

34. Truax, A. D. \& Greer, S. F. ChIP and Re-ChIP assays: investigating interactions between regulatory proteins, histone modifications, and the DNA sequences to which they bind. Methods Mol. Biol. 809, 175-188 (2012).

35. Suzuki, N. et al. Severe impairment of interleukin-1 and Toll-like receptor signalling in mice lacking IRAK-4. Nature 416, 750-756 (2002).

36. Picard, C. et al. Pyogenic bacterial infections in humans with IRAK-4 deficiency. Science 299, 2076-2079 (2003). 
37. Scheinman, R. I., Gualberto, A., Jewell, C. M., Cidlowski, J. A. \& Baldwin, Jr. A. S. Characterization of mechanisms involved in transrepression of NF-kappa B by activated glucocorticoid receptors. Mol. Cell. Biol. 15, 943-953 (1995).

38. Auphan, N., DiDonato, J. A., Rosette, C., Helmberg, A. \& Karin, M. Immunosuppression by glucocorticoids: inhibition of NF-kappa B activity through induction of I kappa B synthesis. Science 270, 286-290 (1995).

39. Kassel, O. et al. Glucocorticoids inhibit MAP kinase via increased expression and decreased degradation of MKP-1. EMBO J. 20, 7108-7116 (2001).

40. Oda, K. \& Kitano, H. A comprehensive map of the toll-like receptor signaling network. Mol. Syst. Biol. 2, 0015 (2006).

41. Li, F., Thiele, I., Jamshidi, N. \& Palsson, B. O. Identification of potential pathway mediation targets in Toll-like receptor signaling. PLoS Comput. Biol. 5, e1000292 (2009).

42. Nissen, R. M. \& Yamamoto, K. R. The glucocorticoid receptor inhibits NFkappaB by interfering with serine- 2 phosphorylation of the RNA polymerase II carboxy-terminal domain. Genes Dev. 14, 2314-2329 (2000).

43. Rao, N. A. et al. Coactivation of GR and NFKB alters the repertoire of their binding sites and target genes. Genome Res. 21, 1404-1416 (2011).

44. Su, J., Xie, Q., Wilson, I. \& Li, L. Differential regulation and role of interleukin1 receptor associated kinase-M in innate immunity signaling. Cell. Signal. 19, 1596-1601 (2007).

45. Cabanski, M. et al. Genome-wide transcriptional profiling of mononuclear phagocytes recruited to mouse lungs in response to alveolar challenge with the TLR2 agonist Pam3CSK4. Am. J. Physiol. Lung Cell. Mol. Physiol. 297, L608-L618 (2009).

46. Nakayama, K. et al. Involvement of IRAK-M in peptidoglycan-induced tolerance in macrophages. J. Biol. Chem. 279, 6629-6634 (2004).

47. Zacharioudaki, V. et al. Adiponectin promotes endotoxin tolerance in macrophages by inducing IRAK-M expression. J. Immunol. 182, 6444-6451 (2009).

48. $\mathrm{Li}, \mathrm{H}$. et al. IL-1 receptor-associated kinase $\mathrm{M}$ is a central regulator of osteoclast differentiation and activation. J. Exp. Med. 201, 1169-1177 (2005).

49. Balaci, L. et al. IRAK-M is involved in the pathogenesis of early-onset persistent asthma. Am. J. Hum. Genet. 80, 1103-1114 (2007).

50. Seki, M. et al. Critical role of IL-1 receptor-associated kinase-M in regulating chemokine-dependent deleterious inflammation in murine influenza pneumonia. J. Immunol. 184, 1410-1418 (2010).

51. van 't Veer, C. et al. Induction of IRAK-M is associated with lipopolysaccharide tolerance in a human endotoxemia model. J. Immunol. 179, 7110-7120 (2007).

52. Medzhitov, R., Schneider, D. S. \& Soares, M. P. Disease tolerance as a defense strategy. Science 335, 936-941 (2012).

53. Tournoy, K. G., Kips, J. C., Schou, C. \& Pauwels, R. A. Airway eosinophilia is not a requirement for allergen-induced airway hyperresponsiveness. Clin. Exp. Allergy 30, 79-85 (2000).

54. Kwak, Y. G. et al. Involvement of PTEN in airway hyperresponsiveness and inflammation in bronchial asthma. J. Clin. Invest. 111, 1083-1092 (2003).

55. Matute-Bello, G. et al. An official American Thoracic Society workshop report: features and measurements of experimental acute lung injury in animals. Am. J. Respir. Cell Mol. Biol. 44, 725-738 (2011).

56. Hu, H. L., Zhang, Q., Kong, B. \& Shi, X. Expression of pancreatic regenerating gene in lung and intestinal tissue correlates with the severity of disease in rats with acute necrotizing pancreatitis. Mol. Med. Rep. 7, 503-508 (2013).
57. Erwin, A. L. \& Smith, A. L. Nontypeable Haemophilus influenzae: understanding virulence and commensal behavior. Trends Microbiol. 15, 355-362 (2007).

58. Yoshida, H., Jono, H., Kai, H. \& Li, J. D. The tumor suppressor cylindromatosis (CYLD) acts as a negative regulator for toll-like receptor 2 signaling via negative cross-talk with TRAF6 AND TRAF7. J. Biol. Chem. 280, 41111-41121 (2005).

59. Lim, J. H. et al. CYLD negatively regulates transforming growth factor-betasignalling via deubiquitinating Akt. Nat. Commun. 3, 771 (2012).

60. Komatsu, K. et al. Inhibition of PDE4B suppresses inflammation by increasing expression of the deubiquitinase CYLD. Nat. Commun. 4, 1684 (2013).

61. Mercurio, F. et al. IKK-1 and IKK-2: cytokine-activated IkappaB kinases essential for NF-kappaB activation. Science 278, 860-866 (1997).

62. Ishinaga, H. et al. TGF-beta induces p65 acetylation to enhance bacteriainduced NF-kappaB activation. EMBO J. 26, 1150-1162 (2007).

63. Johannessen, C. M. et al. COT drives resistance to RAF inhibition through MAP kinase pathway reactivation. Nature 468, 968-972 (2010).

64. Wang, J. C. et al. Chromatin immunoprecipitation (ChIP) scanning identifies primary glucocorticoid receptor target genes. Proc. Natl Acad. Sci. USA 101, 15603-15608 (2004).

\section{Acknowledgements}

This work was supported by grants from the National Institutes of Health DC005843, DC004562 and GM107529 to J.D.L. J.D.L. is a Georgia Research Alliance Eminent Scholar in Inflammation and Immunity. R.A.F. is a Howard Hughes Medical Institute (HHMI) investigator.

\section{Author contributions}

M.M., J.-Y.L., S.S.-M., H.X. and J.-D.L. designed the experiments and analysed the data. M.M., J.-Y.L. and S.S.-M. performed the experiments. H.K. contributed to data analysis and discussion. R.A.F. and K.S.K. contributed materials and also contributed to discussion. M.M., W.Y.W. and J.-D.L. wrote the manuscript.

\section{Additional information}

Supplementary Information accompanies this paper at http://www.nature.com/ naturecommunications

Competing financial interests: The authors declare no competing financial interests.

Reprints and permission information is available online at http://npg.nature.com/ reprintsandpermissions/

How to cite this article: Miyata, M. et al. Glucocorticoids suppress inflammation via the upregulation of negative regulator IRAK-M. Nat. Commun. 6:6062 doi: $10.1038 /$ ncomms7062 (2015).

(c) (i) This work is licensed under a Creative Commons Attribution 4.0 International License. The images or other third party material in this article are included in the article's Creative Commons license, unless indicated otherwise in the credit line; if the material is not included under the Creative Commons license, users will need to obtain permission from the license holder to reproduce the material. To view a copy of this license, visit http://creativecommons.org/licenses/by/4.0/ 